\title{
Chiral perturbation theory analysis of the quark condensate in a magnetic field
}

\author{
Christoph P. Hofmann \\ Facultad de Ciencias, Universidad de Colima, Bernal Díaz del Castillo 340, Colima C.P. 28045, Mexico
}

(Received 22 June 2020; accepted 20 October 2020; published 12 November 2020)

\begin{abstract}
We present two-loop results for the quark condensate in an external magnetic field within chiral perturbation theory using coordinate-space techniques. At finite temperature, we explore the impact of the magnetic field on the pion-pion interaction in the quark condensate for arbitrary pion masses and derive the correct weak magnetic field expansion in the chiral limit. At zero temperature, we provide the complete two-loop representation for the vacuum energy density and the quark condensate.
\end{abstract}

DOI: 10.1103/PhysRevD.102.094010

\section{INTRODUCTION}

The quark condensate — order parameter of spontaneous chiral symmetry breaking - is a crucial quantity in particle physics. It comes with no surprise that the relevant literature is extensive. Here we focus on the properties of the quark condensate in an external constant magnetic field. Our calculation within the framework of chiral perturbation theory (CHPT) goes up to two-loop order, but in contrast to the available CHPT studies-see Refs. [1-10]—we use a novel representation for the kinematical functions that we established in Ref. [11]. Other references, also dealing with the quark condensate in a magnetic field, are based upon lattice QCD [12-21], feature analytical studies relying on the Nambu-JonaLasinio model [22-29], or comprise yet other models and methods [30-37].

In a recent article [11], the present author has pointed out that - in the chiral limit - the two published one-loop series for the finite-temperature quark condensate in a weak magnetic field, independently derived by different authors, are erroneous. The proper series at one-loop order has been established in Ref. [11] - one of our goals in the actual study is to review the situation at two-loop order. Indeed, errors also occur here. We clarify the situation by providing the correct weak magnetic field expansion of the finite-temperature quark condensate in the chiral limit. One of the advantages of our coordinate-space approach is that it allows for a transparent

Published by the American Physical Society under the terms of the Creative Commons Attribution 4.0 International license. Further distribution of this work must maintain attribution to the author(s) and the published article's title, journal citation, and DOI. Funded by SCOAP . derivation of the various limits that have to be taken in the calculation: chiral limit $\left(M_{\pi} \rightarrow 0\right)$ and weak magnetic field limit $\left(|q H| \ll T^{2}\right){ }^{1}$

Apart from straightening these issues, we also investigate the impact of the magnetic field on the pion-pion interaction in the quark condensate for arbitrary pion masses. At finite temperature, the interaction constitutes up to $10 \%$ as compared to the leading noninteracting pion gas contribution and is most pronounced in the chiral limit. When the magnetic field increases, the finite-temperature quark condensate (sum of one- and two-loop contributions at fixed temperature and pion mass) grows monotonically. The effect is again most pronounced in the chiral limit.

Using the dressed pions as pertinent degrees of freedom, the low-temperature series of the quark condensate is characterized by a $T^{2}$ contribution that refers to the dressed but noninteracting pions, while interaction effects emerge at order $T^{4}$. In the chiral limit and in weak magnetic fields, the series at order $T^{2}$-organized by the expansion parameter $\epsilon=|q H| / T^{2}$ ( $q$ is the electric charge of the pion) —involves a leading square-root term $\propto \sqrt{\epsilon}$, a term linear in $\epsilon$, followed by a half-integer power $\epsilon^{3 / 2}$ and a logarithmic contribution $\epsilon^{2} \ln \epsilon$. The remaining contributions involve even powers of $\epsilon$. At order $T^{4}$ the series exhibits the same structure, with the exception that a term linear in $\epsilon$ is absent-in contrast to what has been reported in the literature.

\footnotetext{
${ }^{1}$ It should be noted that there are three different scales in play: pion mass, magnetic field strength and temperature. The chiral limit corresponds to taking the limit $M_{\pi} \rightarrow 0$ at fixed temperature. Accordingly, the theory in the chiral limit is then characterized by only two scales: magnetic field strength $(|q H|)$ and $T$. In this situation we can then take another limit- $|q H| \ll T^{2}$ - that we call "weak magnetic field limit," where "weak" means magnetic field strength small with respect to temperature. This latter limit thus always refers to the situation where the chiral limit at fixed temperature has been taken first.
} 
Finally, we provide the two-loop representation for the QCD vacuum energy density and the zero-temperature quark condensate. The representation involves nonanalytic contributions in the form of logarithms, as well as Gamma and polygamma functions that depend nontrivially on the ratio between magnetic field and pion mass. In contrast to previous studies we provide the full two-loop representation-not merely the terms that are induced by the nonzero magnetic field.

The article is organized as follows. The two-loop CHPT evaluation is briefly reviewed in Sec. II to set the basis for the subsequent analysis. In Sec. III we explore the quark condensate at finite and zero temperature for arbitrary pion masses - in particular also for the physical pion masses - in the presence of a magnetic field. In the same section we furthermore compare our findings with the literature and point out errors in the published results. Finally, Sec. IV contains our conclusions. More technical issues are presented in three Appendixes. In Appendix A we discuss in detail the two-loop CHPT evaluation at zero temperature. While Appendix B is devoted to the chiral limit in nonzero magnetic fields at $T=0$, in Appendix $\mathrm{C}$ we consider the same situation at finite temperature which boils down to an analysis of the various kinematical functions required.

\section{CHIRAL PERTURBATION THEORY EVALUATION}

The relevant low-energy excitations in two-flavor chiral perturbation theory ${ }^{2}$ are the three pions that are incorporated in the $\mathrm{SU}(2)$ matrix $U(x)$ as

$$
U(x)=\exp \left(i \tau^{i} \pi^{i}(x) / F\right), \quad i=1,2,3 .
$$

Here $\tau^{i}$ are the Pauli matrices and $F$ stands for the tree-level pion decay constant. While $\pi^{0}$ describes the neutral pion, ${ }^{3}$ the charged pions correspond to the linear combinations

$$
\pi^{ \pm}=\frac{1}{\sqrt{2}}\left(\pi^{1} \pm i \pi^{2}\right)
$$

The Euclidean leading-order (order $p^{2}$ ) effective Lagrangian is given by

$$
\mathcal{L}_{\text {eff }}^{2}=\frac{1}{4} F^{2} \operatorname{Tr}\left[\left(D_{\mu} U\right)^{\dagger}\left(D_{\mu} U\right)-M^{2}\left(U+U^{\dagger}\right)\right],
$$

where $M$ is the tree-level pion mass. The covariant derivative is defined as [see Eq. (4.58) in Ref. [39] ]

\footnotetext{
${ }^{2}$ For reviews of chiral perturbation theory see, e.g., Refs. [38,39].

${ }^{3}$ Although the Pauli matrix associated with the neutral pion is $\tau^{3}$, we will denote the neutral pion field as $\pi^{0}$ in view of its zero charge.

${ }^{4}$ In Refs. [11,40] the covariant derivative was not displayed correctly. The error concerns Eq. (3.4) in Ref. [11] and Eq. (2.3) in Ref. [40]. We emphasize that all derivations involving the covariant derivative in Refs. [11,40] were based on the correct expression Eq. (2.4) displayed here - no further corrections or modifications in any previous articles of the present author are necessary.
}

$$
D_{\mu} U=\partial_{\mu} U-i r_{\mu} U+i U l_{\mu} .
$$

In two-flavor QCD the external electromagnetic field appears in the left- and right-handed currents as [see Eq. (2.111) in Ref. [39] ]

$$
r_{\mu}=l_{\mu}=-\frac{e}{2} \tau_{3} A_{\mu}^{\mathrm{EM}} .
$$

The quantity $e$ is the electric charge, while the magnetic field $H$ enters via the gauge field

$$
A_{\mu}^{\mathrm{EM}}=\left(0,0, H x_{1}, 0\right) .
$$

As illustrated in Fig. 1, a two-loop calculation of the free energy density in addition involves the subleading pieces $\mathcal{L}_{\text {eff }}^{4}$ and $\mathcal{L}_{\text {eff }}^{6}$ of the effective Lagrangian.

The set of terms proportional to four pion fields generated by the leading piece $\mathcal{L}_{\text {eff }}^{2}$-as required for the evaluation of the two-loop diagram $6 A$-are

$$
\begin{aligned}
\mathcal{L}_{\{4\}}^{2}= & \frac{1}{3 F^{2}} \pi^{0} \partial_{\mu} \pi^{0}\left(\partial_{\mu} \pi^{+} \pi^{-}+\partial_{\mu} \pi^{-} \pi^{+}\right) \\
& -\frac{1}{3 F^{2}} \partial_{\mu} \pi^{0} \partial_{\mu} \pi^{0} \pi^{+} \pi^{-}-\frac{1}{3 F^{2}} \pi^{0} \pi^{0} \partial_{\mu} \pi^{+} \partial_{\mu} \pi^{-} \\
& -\frac{1}{3 F^{2}} \pi^{+} \pi^{-} \partial_{\mu} \pi^{+} \partial_{\mu} \pi^{-} \\
& +\frac{1}{6 F^{2}}\left(\partial_{\mu} \pi^{+} \pi^{-} \partial_{\mu} \pi^{+} \pi^{-}+\partial_{\mu} \pi^{-} \pi^{+} \partial_{\mu} \pi^{-} \pi^{+}\right) .
\end{aligned}
$$

Other pieces from $\mathcal{L}_{\text {eff }}^{2}$ needed for our calculation are terms with two (diagram $4 A$ ) or zero (diagram 2) pion fields,

$\mathcal{L}_{\{2\}}^{2}=\frac{1}{2} \partial_{\mu} \pi^{0} \partial_{\mu} \pi^{0}+\partial_{\mu} \pi^{+} \partial_{\mu} \pi^{-}+\frac{1}{2} M^{2} \pi^{0} \pi^{0}+M^{2} \pi^{+} \pi^{-}$, $\mathcal{L}_{\{0\}}^{2}=-F^{2} M^{2}$.

As for the subleading piece $\mathcal{L}_{\text {eff }}^{4}$, we use the representation given in Eq. (D.2) of Ref. [39]. The relevant terms for our calculation are those that contain two (diagram $6 B$ ) or zero (diagram $4 B$ ) pion fields:

$\mathcal{L}_{\{2\}}^{4}=l_{3} \frac{M^{4}}{F^{2}} \pi^{0} \pi^{0}+2 l_{3} \frac{M^{4}}{F^{2}} \pi^{+} \pi^{-}+\left(4 l_{5}-2 l_{6}\right) \frac{|q H|^{2}}{F^{2}} \pi^{+} \pi^{-}$,

$\mathcal{L}_{\{0\}}^{4}=-\left(l_{3}+h_{1}\right) M^{4}+4 h_{2}|q H|^{2}$.

The quantities $l_{3}, l_{5}, l_{6}, h_{1}$, and $h_{2}$ are next-to-leading-order (NLO) low-energy effective constants.

Finally, following Ref. [41], the terms from $\mathcal{L}_{\text {eff }}^{6}$ contributing to the tree-level diagram $6 C$ read

$$
\mathcal{L}_{\{0\}}^{6}=-16\left(c_{10}+2 c_{11}\right) M^{6}-8 c_{34} M^{2}|q H|^{2},
$$

where $c_{10}, c_{11}$, and $c_{34}$ are next-to-next-to-leading-order (NNLO) low-energy effective constants. 


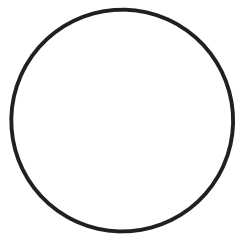

$4 A$

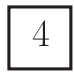

$4 B$

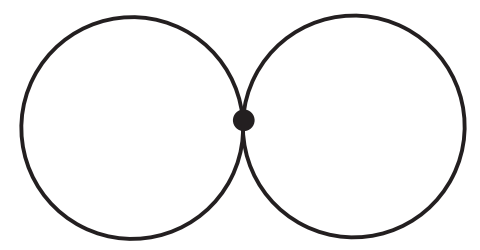

$6 A$

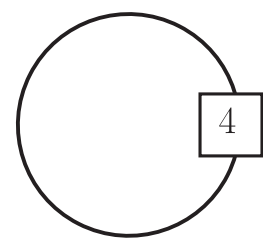

$6 B$

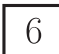

$6 C$

FIG. 1. Chiral perturbation theory diagrams for the QCD free energy density up to order $p^{6}$. Vertices from $\mathcal{L}_{\text {eff }}^{2}$ (filled circles), as well as vertices from $\mathcal{L}_{\text {eff }}^{4}$ and $\mathcal{L}_{\text {eff }}^{6}$ (denoted by the numbers 4 and 6, respectively) contribute. The lines refer to the thermal pion propagators.

As we show in Appendix A 1, all effective low-energy constants become singular in the physical limit $d \rightarrow 4$, and the divergence is contained in the parameter $\lambda$ :

$$
\begin{aligned}
\lambda & =\frac{1}{2}(4 \pi)^{-d / 2} \Gamma\left(1-\frac{1}{2} d\right) \mu^{d-4} \\
& =\frac{\mu^{d-4}}{16 \pi^{2}}\left[\frac{1}{d-4}-\frac{1}{2}\left\{\ln 4 \pi+\Gamma^{\prime}(1)+1\right\}+\mathcal{O}(d-4)\right],
\end{aligned}
$$

where $\mu$ is the scale of dimensional regularization. While the NLO effective constants $l_{3}, l_{5}, l_{6}, h_{1}$, and $h_{2}$ and the NNLO effective constant $c_{34}$ are linear in $\lambda$ [see Eqs. (A3) and (A11)], the NNLO effective constants $c_{10}$ and $c_{11}$ in addition involve divergences quadratic in $\lambda$ [see Eq. (A18)]. These ultraviolet divergences exactly cancel the $\lambda$ divergences that also emerge in the zero-temperature propagators $\Delta^{ \pm}(0)$ and $\Delta^{0}(0)$-Eq. (A27)-which constitute the $T=0$ piece in the thermal propagators according to Eq. (A26). As a consequence, the sum of all contributions in the two-loop free energy density is finite. While the renormalization procedure for the $T=0$ case is outlined in detail in Appendix A 2, the renormalization of the finitetemperature piece in the free energy density is discussed at length in Appendix A of Ref. [40].

It is convenient to divide the free energy density into two pieces as

$$
z=z_{0}+z^{T},
$$

where $z_{0}$ contains all $T=0$ contributions (vacuum energy density) and $z^{T}$ involves the finite-temperature part-both terms depend on the magnetic field. Before addressing the $T=0$ case, we quote the result for the finite-temperature piece which has been derived within the CHPT coordinatespace approach up to two-loop order in Ref. [40]:

$$
\begin{aligned}
z^{T}= & -g_{0}\left(M_{\pi}^{ \pm}, T, 0\right)-\frac{1}{2} g_{0}\left(M_{\pi}^{0}, T, 0\right)-\tilde{g}_{0}\left(M_{\pi}^{ \pm}, T, H\right) \\
& +\frac{M_{\pi}^{2}}{2 F^{2}} g_{1}\left(M_{\pi}^{ \pm}, T, 0\right) g_{1}\left(M_{\pi}^{0}, T, 0\right)-\frac{M_{\pi}^{2}}{8 F^{2}}\left\{g_{1}\left(M_{\pi}^{0}, T, 0\right)\right\}^{2} \\
& +\frac{M_{\pi}^{2}}{2 F^{2}} g_{1}\left(M_{\pi}^{0}, T, 0\right) \tilde{g}_{1}\left(M_{\pi}^{ \pm}, T, H\right)+\mathcal{O}\left(p^{8}\right) .
\end{aligned}
$$

The kinematical Bose functions are defined as ${ }^{5}$

$$
\begin{aligned}
g_{0}(\mathcal{M}, T, 0)= & T^{4} \int_{0}^{\infty} \mathrm{d} \rho \rho^{-3} \exp \left(-\frac{\mathcal{M}^{2}}{4 \pi T^{2}} \rho\right)\left[S\left(\frac{1}{\rho}\right)-1\right], \\
g_{1}(\mathcal{M}, T, 0)= & \frac{T^{2}}{4 \pi} \int_{0}^{\infty} \mathrm{d} \rho \rho^{-2} \exp \left(-\frac{\mathcal{M}^{2}}{4 \pi T^{2}} \rho\right)\left[S\left(\frac{1}{\rho}\right)-1\right], \\
\tilde{g}_{0}\left(M_{\pi}^{ \pm}, T, H\right)= & \frac{T^{2}}{4 \pi}|q H| \int_{0}^{\infty} \mathrm{d} \rho \rho^{-2} \\
& \times\left(\frac{1}{\sinh \left(|q H| \rho / 4 \pi T^{2}\right)}-\frac{4 \pi T^{2}}{|q H| \rho}\right) \\
& \times \exp \left(-\frac{\left(M_{\pi}^{ \pm}\right)^{2}}{4 \pi T^{2}} \rho\right)\left[S\left(\frac{1}{\rho}\right)-1\right], \\
\tilde{g}_{1}\left(M_{\pi}^{ \pm}, T, H\right)= & \frac{1}{16 \pi^{2}}|q H| \int_{0}^{\infty} \mathrm{d} \rho \rho^{-1} \\
& \times\left(\frac{1}{\sinh \left(|q H| \rho / 4 \pi T^{2}\right)}-\frac{4 \pi T^{2}}{|q H| \rho}\right) \\
& \times \exp \left(-\frac{\left(M_{\pi}^{ \pm}\right)^{2}}{4 \pi T^{2}} \rho\right)\left[S\left(\frac{1}{\rho}\right)-1\right],
\end{aligned}
$$

and $S(z)$ stands for the Jacobi theta function:

$$
S(z)=\sum_{n=-\infty}^{\infty} \exp \left(-\pi n^{2} z\right)
$$

Note that $\tilde{g}_{0}$ and $\tilde{g}_{1}$ explicitly depend on the magnetic field through the hyperbolic sine and that they involve the mass $M_{\pi}^{ \pm}$, i.e., the masses of the charged pions in a magnetic field given by

$$
\left(M_{\pi}^{ \pm}\right)^{2}=M_{\pi}^{2}+\frac{\bar{l}_{6}-\bar{l}_{5}}{48 \pi^{2}} \frac{|q H|^{2}}{F^{2}} .
$$

The mass $\mathcal{M}$ in $g_{0}$ and $g_{1}$, according to Eq. (2.13), can either represent $M_{\pi}^{ \pm}$or $M_{\pi}^{0}$, where the latter is the mass of the neutral pion in a magnetic field,

\footnotetext{
${ }^{5}$ The advantage of the novel kinematical functions $\tilde{g}_{0}$ and $\tilde{g}_{1}$ is that in the chiral limit they allow for a clear-cut expansion in terms of the parameter $|q H| / T^{2}$; i.e., they provide the basis for a transparent analysis of the quark condensate in the weak magnetic field limit, where other authors have failed (see Refs. $[3-5,9,10]$ ). The explicit construction of these functions can be found in Ref. [11]—see Eqs. (3.14)-(3.19).
} 


$$
\left(M_{\pi}^{0}\right)^{2}=M_{\pi}^{2}+\frac{M^{2}}{F^{2}} K_{1}
$$

and $K_{1}$ denotes the integral

$K_{1}=\frac{|q H|}{16 \pi^{2}} \int_{0}^{\infty} \mathrm{d} \rho \rho^{-1} \exp \left(-\frac{M_{\pi}^{2}}{|q H|} \rho\right)\left(\frac{1}{\sinh (\rho)}-\frac{1}{\rho}\right)$.

The kinematical functions $g_{0}$ and $g_{1}$ hence implicitly depend on the magnetic field through the neutral and charged pion masses. Finally, the mass $M_{\pi}$ is the renormalized NLO pion mass in zero magnetic field:

$$
M_{\pi}^{2}=M^{2}-\frac{\bar{l}_{3}}{32 \pi^{2}} \frac{M^{4}}{F^{2}}+\mathcal{O}\left(M^{6}\right) .
$$

The quantities $\bar{l}_{3}, \bar{l}_{5}$, and $\bar{l}_{6}$ are renormalized NLO low-energy effective constants-details are provided in Appendix A 1.

We now address the zero-temperature part in the free energy density. ${ }^{6}$ Apart from the temperature-independent tree-level graphs $2,4 B$ and $6 C$, we also have $T=0$ contributions from the loop graphs. This is because the thermal propagators for the pions,

$$
\begin{aligned}
G^{ \pm}(x) & =\sum_{n=-\infty}^{\infty} \Delta^{ \pm}\left(\vec{x}, x_{4}+n \beta\right), \\
G^{0}(x) & =\sum_{n=-\infty}^{\infty} \Delta^{0}\left(\vec{x}, x_{4}+n \beta\right), \quad \beta=\frac{1}{T},
\end{aligned}
$$

contain a zero-temperature piece associated with $n=0$. In Appendix A 2 we process these $T=0$ contributions and show that all UV divergences cancel. The final result for the renormalized vacuum energy density at order $p^{6}$ then amounts to

$$
\begin{aligned}
z_{0}^{[6]}= & \frac{3 \bar{l}_{3}\left(\bar{c}_{10}+2 \bar{c}_{11}\right)}{1024 \pi^{4}} \frac{M^{6}}{F^{2}}-\frac{\left(\bar{l}_{6}-\bar{l}_{5}\right) \bar{c}_{34}}{768 \pi^{4}} \frac{|q H|^{2} M^{2}}{F^{2}} \\
& -\frac{\bar{l}_{3}}{32 \pi^{2}} \frac{M^{4}}{F^{2}} K_{1}+\frac{\left(\bar{l}_{6}-\bar{l}_{5}\right)}{48 \pi^{2}} \frac{|q H|^{2}}{F^{2}} K_{1} .
\end{aligned}
$$

The quantities $\bar{l}_{i}$ and $\bar{c}_{i}$ are the renormalized NLO and NNLO effective constants, respectively, defined in Appendix A 1.

The full vacuum energy density also includes the zerotemperature pieces of order $p^{4}$ and $p^{2}$ :

\footnotetext{
${ }^{6}$ To the best of our knowledge, the complete CHPT two-loop representation for the QCD vacuum energy density-containing magnetic-field-dependent as well as $H$-independent terms-is not available in the literature.
}

$$
z_{0}=z_{0}^{[6]}+z_{0}^{[4]}+z_{0}^{[2]}
$$

which are (see Ref. [11] for $z_{0}^{[4]}$ )

$$
\begin{aligned}
z_{0}^{[4]}= & \frac{M^{4}}{64 \pi^{2}}\left(\bar{l}_{3}-4 \bar{h}_{1}-\frac{3}{2}\right)+\frac{|q H|^{2}}{96 \pi^{2}}\left(\bar{h}_{2}-1\right) \\
& -\frac{|q H|^{2}}{16 \pi^{2}} \int_{0}^{\infty} \mathrm{d} \rho \rho^{-2}\left(\frac{1}{\sinh (\rho)}-\frac{1}{\rho}+\frac{\rho}{6}\right) \exp \left(-\frac{M^{2}}{|q H|} \rho\right),
\end{aligned}
$$

$z_{0}^{[2]}=-F^{2} M^{2}$.

The subleading contributions $z_{0}^{[4]}$ and $z_{0}^{[6]}$ as displayed above, i.e., the renormalized expressions, are independent of the renormalization scale $\mu$. This is a nontrivial consistency check of our calculation. We now turn to the quark condensate which is the main subject of the present investigation.

\section{QUARK CONDENSATE IN A MAGNETIC FIELD}

The quark condensate is given by the derivative of the free energy density with respect to the quark mass ${ }^{7}$

$$
\langle\bar{q} q\rangle=\frac{\mathrm{d} z}{\mathrm{~d} m_{q}} .
$$

At zero temperature it corresponds to the vacuum expectation value

$$
\langle 0|\bar{q} q| 0\rangle=\frac{\mathrm{d} z_{0}}{\mathrm{~d} m_{q}}=-\frac{\langle 0|\bar{q} q| 0\rangle_{0}}{F^{2}} \frac{\mathrm{d} z_{0}}{\mathrm{~d} M^{2}}
$$

Note that we have used the leading-order Gell-MannOakes-Renner relation

$$
M^{2}=-\frac{m_{q}}{F^{2}}\langle 0|\bar{q} q| 0\rangle_{0},
$$

where the quantity $\langle 0|\bar{q} q| 0\rangle_{0}$ is the quark condensate at $T=0$ (and zero magnetic field) in the chiral limit—as indicated by the lower index " 0 ." The purely finite-temperature part in the quark condensate amounts to

$$
\langle\bar{q} q\rangle^{T}=-\frac{\mathrm{d} P}{\mathrm{~d} m_{q}}=\frac{\langle 0|\bar{q} q| 0\rangle_{0}}{F^{2}} \frac{\mathrm{d} P}{\mathrm{~d} M^{2}} .
$$

Up to the sign, the pressure is nothing but the finitetemperature piece in the free energy density,

$$
P=-z^{T}
$$

\footnotetext{
${ }^{7}$ Throughout the study we work in the isospin limit $m_{q}=$ $m_{u}=m_{d}$.
} 
In the representation of $z^{T}$ [Eq. (2.13)], we have used the NLO renormalized pion mass $M_{\pi}$ instead of $M$. The connection between the two quantities is given by Eq. (2.19):

$$
M_{\pi}^{2}=M^{2}-\frac{\bar{l}_{3}}{32 \pi^{2}} \frac{M^{4}}{F^{2}}+\mathcal{O}\left(M^{6}\right) .
$$

For the quark condensate we then obtain

$$
\langle\bar{q} q\rangle=\frac{\langle 0|\bar{q} q| 0\rangle_{0}}{F^{2}}\left\{-\frac{\mathrm{d} z_{0}}{\mathrm{~d} M_{\pi}^{2}}+\frac{\mathrm{d} P}{\mathrm{~d} M_{\pi}^{2}}\right\}\left(1-\frac{M_{\pi}^{2}}{32 \pi^{2} F^{2}}\left(2 \bar{l}_{3}-1\right)\right) .
$$

Note that the large parenthesis corresponds to the correction $\mathrm{d} M_{\pi}^{2} / \mathrm{d} M^{2}$. In particular, the " 1 " in the small parenthesis $\left(2 \bar{l}_{3}-1\right)$ appears because the NLO constant $\bar{l}_{3}$ depends on the pion mass as (see Ref. [42])

$$
\frac{\mathrm{d} \bar{l}_{i}}{\mathrm{~d} M^{2}}=-\frac{1}{M^{2}}
$$

After these manipulations, in the large parenthesis we can then replace $M^{2}$ by $M_{\pi}^{2}$ which is legitimate at the order we are operating.

It should be pointed out that the zero-temperature quark condensate at order $p^{4}$, according to Eq. (2.23), involves the NLO effective constant $\bar{h}_{1}$ which depends on the renormalization convention (see Ref. [42]). No such ambiguities due to NLO effective constants $\bar{h}_{i}$ are introduced in the zero-temperature quark condensate at order $p^{6}$, according to Eq. (2.21). Likewise, the finitetemperature part of the quark condensate is also free of such renormalization ambiguities.

\section{A. Finite-temperature quark condensate}

In order to make powers of temperature in the quark condensate manifest, instead of operating with the Bose functions $g_{r}$ and $\tilde{g}_{r}$, we now work with the dimensionless functions $h_{r}$ and $\tilde{h}_{r}$ defined as

$$
\begin{array}{lll}
h_{0}=\frac{g_{0}}{T^{4}}, & \tilde{h}_{0}=\frac{\tilde{g}_{0}}{T^{4}}, & h_{1}=\frac{g_{1}}{T^{2}}, \\
\tilde{h}_{1}=\frac{\tilde{g}_{1}}{T^{2}}, & h_{2}=g_{2}, & \tilde{h}_{2}=\tilde{g}_{2} .
\end{array}
$$

With the expression for $z^{T}$ [Eq. (2.13)], the finite-temperature part of the quark condensate takes the form

$$
\begin{aligned}
& \frac{\langle\bar{q} q\rangle^{T}}{\langle 0|\bar{q} q| 0\rangle_{0}}\left(1-\frac{M_{\pi}^{2}}{32 \pi^{2} F^{2}}\left(2 \bar{l}_{3}-1\right)\right)^{-1} \\
& =-\left\{\frac{q_{1}}{F^{2}} T^{2}+\frac{q_{2}}{F^{4}} T^{4}+\mathcal{O}\left(T^{6}\right)\right\} .
\end{aligned}
$$

The respective coefficients

$$
\begin{aligned}
q_{1}= & h_{1}\left(M_{\pi}^{ \pm}, T, 0\right)+\frac{1}{2} a_{0} h_{1}\left(M_{\pi}^{0}, T, 0\right)+\tilde{h}_{1}\left(M_{\pi}^{ \pm}, T, H\right), \\
q_{2}= & +\frac{1}{2} h_{1}\left(M_{\pi}^{ \pm}, T, 0\right) h_{1}\left(M_{\pi}^{0}, T, 0\right)+\frac{1}{2} h_{1}\left(M_{\pi}^{0}, T, 0\right) \tilde{h}_{1}\left(M_{\pi}^{ \pm}, T, H\right) \\
& -\frac{1}{8} h_{1}\left(M_{\pi}^{0}, T, 0\right) h_{1}\left(M_{\pi}^{0}, T, 0\right)-\frac{1}{2} \frac{m^{2}}{t^{2}} h_{1}\left(M_{\pi}^{0}, T, 0\right) h_{2}\left(M_{\pi}^{ \pm}, T, 0\right) \\
& -\frac{1}{2} a_{0} \frac{m^{2}}{t^{2}} h_{1}\left(M_{\pi}^{ \pm}, T, 0\right) h_{2}\left(M_{\pi}^{0}, T, 0\right)-\frac{1}{2} a_{0} \frac{m^{2}}{t^{2}} \tilde{h}_{1}\left(M_{\pi}^{ \pm}, T, H\right) h_{2}\left(M_{\pi}^{0}, T, 0\right) \\
& +\frac{1}{4} a_{0} \frac{m^{2}}{t^{2}} h_{1}\left(M_{\pi}^{0}, T, 0\right) h_{2}\left(M_{\pi}^{0}, T, 0\right)-\frac{1}{2} \frac{m^{2}}{t^{2}} h_{1}\left(M_{\pi}^{0}, T, 0\right) \tilde{h}_{2}\left(M_{\pi}^{ \pm}, T, H\right)
\end{aligned}
$$

depend in a nontrivial way on the ratios between temperature, pion masses and magnetic field. Instead of the three dimensionful quantities $T, M_{\pi}$ and $|q H|$, we now use the dimensionless parameters $t, m$, and $m_{H}$ defined, respectively, as

$$
t=\frac{T}{4 \pi F}, \quad m=\frac{M_{\pi}}{4 \pi F}, \quad m_{H}=\frac{\sqrt{|q H|}}{4 \pi F} .
$$

The NLO mass correction $a_{0}$ in the coefficient $q_{2}$ of Eq. (3.10) is

$$
a_{0}=\frac{\mathrm{d}\left(M_{\pi}^{0}\right)^{2}}{\mathrm{~d} M_{\pi}^{2}}=1+\frac{K_{1}}{F^{2}}+\frac{M_{\pi}^{2}}{F^{2}} \frac{\mathrm{d} K_{1}}{\mathrm{~d} M_{\pi}^{2}},
$$

with the integral $\mathrm{d} K_{1} / \mathrm{d} M_{\pi}^{2}$ given by

$\frac{\mathrm{d} K_{1}}{\mathrm{~d} M_{\pi}^{2}}=-\frac{1}{16 \pi^{2}} \int_{0}^{\infty} \mathrm{d} \rho \exp \left(-\frac{M_{\pi}^{2}}{|q H|} \rho\right)\left(\frac{1}{\sinh (\rho)}-\frac{1}{\rho}\right)$.

The coefficient $q_{1}$ refers to the free pion gas contribution of order $T^{2}$, while the coefficient $q_{2}$ captures the pion-pion 

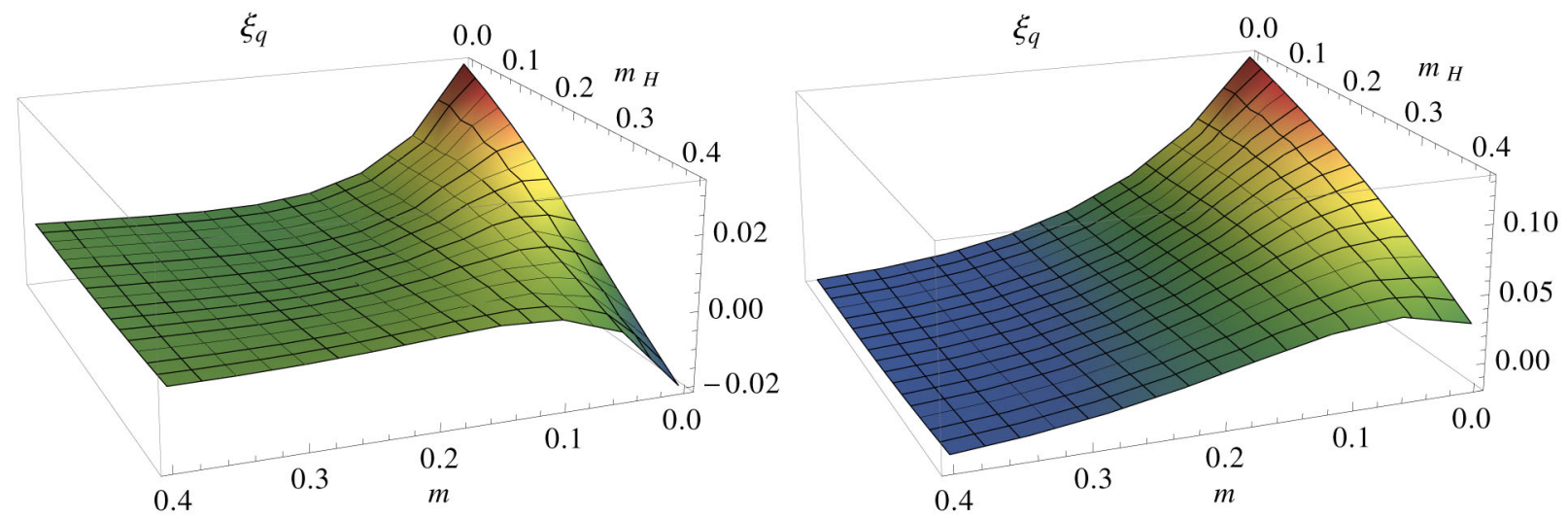

FIG. 2. Magnitude and sign of the pion-pion interaction in the finite-temperature quark condensate measured by $\xi_{q}\left(t, m, m_{H}\right)-$ Eq. (3.14)—referring to the temperatures $T=108 \mathrm{MeV}$ (left) and $T=215 \mathrm{MeV}$ (right).

interaction that emerges at order $T^{4}$ in the finitetemperature quark condensate.

To assess the magnitude of the interaction, in Fig. 2, we plot the dimensionless ratio

$$
\xi_{q}\left(t, m, m_{H}\right)=\frac{q_{2} T^{2}}{q_{1} F^{2}}
$$

that measures the effect of the pion-pion interaction in the quark condensate relative to the free pion gas contribution. The dimensionless quantities $t, m$, and $m_{H}$-defined in Eq. (3.11) — capture, respectively, the temperature, pion mass, and strength of the magnetic field relative to the chiral symmetry-breaking scale $\Lambda_{\chi} \approx 4 \pi F \approx 1 \mathrm{GeV}$. The quantities $t, m$, and $m_{H}$ must be small since chiral perturbation theory is a low-energy effective theory.

In Fig. 2 we have chosen $T=108 \mathrm{MeV}$ and $T=$ $215 \mathrm{MeV}$ (or $t=\{0.1,0.2\}$ ) as well as $m, m_{H} \leq 0.4$. One observes that the interaction is largest in the chiral limit $(m \rightarrow 0)$. At lower temperatures, such as $T=$ $108 \mathrm{MeV}$, the interaction correction may be positive (when the magnetic field is weak) or negative (when the magnetic field is stronger). At more elevated temperatures, such as $T=215 \mathrm{MeV}$, the interaction correction in the parameter domain $m, m_{H} \leq 0.4$ is strictly positive and largest when the magnetic field is turned off. Notice that the effect of the interaction is not tiny-rather it may constitute up to about $13 \%$ relative to the leading free pion gas contribution.

In Fig. 3, we depict the sum of one- and two-loop contributions, i.e., the dimensionless quantity

$$
-\left(q_{1}+q_{2} \frac{T^{2}}{F^{2}}\right)
$$

for the same two temperatures $T=\{108 \mathrm{MeV}, 215 \mathrm{MeV}\}$, or $t=\{0.1,0.2\}$. As the plots indicate-at fixed $M_{\pi}$ and temperature - the finite-temperature quark condensate increases when the magnetic field grows. The effect is most pronounced in the chiral limit $(m \rightarrow 0)$.

Let us examine the real world, where the pion masses are fixed at their physical values $M_{\pi}=140 \mathrm{MeV}$ $(m=0.130){ }^{8}$ In Fig. 4 , on the lhs, we plot the ratio $\xi_{q}$ as a function of temperature and magnetic field strength. The effect of the pion-pion interaction is less than $10 \%$ in the parameter range $t, m_{H} \leq 0.25(T \leq 269 \mathrm{MeV}$, $\sqrt{|q H|} \leq 269 \mathrm{MeV}$ ) we are considering. Finally, on the rhs of Fig. 4, we depict the sum of one- and two-loop contributions in the quark condensate for the same parameter domain. One observes that the finite-temperature quark condensate slightly increases when the strength of the magnetic field grows while temperature is held constant. This effect however is small.

In the chiral limit, the finite-temperature quark condensate reduces to

$$
\begin{aligned}
\frac{\langle\bar{q} q\rangle^{T}}{\langle 0|\bar{q} q| 0\rangle_{0}}= & -\frac{T^{2}}{F^{2}}\left\{\frac{1}{24}+h_{1}\left(M_{H}, T, 0\right)\right. \\
& \left.-\left(\frac{|q H| \ln 2}{32 \pi^{2} F^{2}}\right) h_{1}(0, T, 0)+\tilde{h}_{1}\left(M_{H}, T, H\right)\right\} \\
& +\frac{T^{4}}{24 F^{4}}\left\{\frac{1}{48}-h_{1}\left(M_{H}, T, 0\right)-\tilde{h}_{1}\left(M_{H}, T, H\right)\right\} \\
& +\mathcal{O}\left(T^{6}\right) .
\end{aligned}
$$

The mass $M_{H}$ depends on the magnetic field,

$$
M_{H}^{2}=\frac{\bar{l}_{6}-\bar{l}_{5}}{48 \pi^{2}} \frac{|q H|^{2}}{F^{2}}
$$

\footnotetext{
${ }^{8}$ For the tree-level pion decay constant we use the value $F=85.6 \mathrm{MeV}$ reported in Ref. [43]. Note that in the isospin limit - and in zero magnetic field - the masses of the neutral and the charged pions are identical.
} 

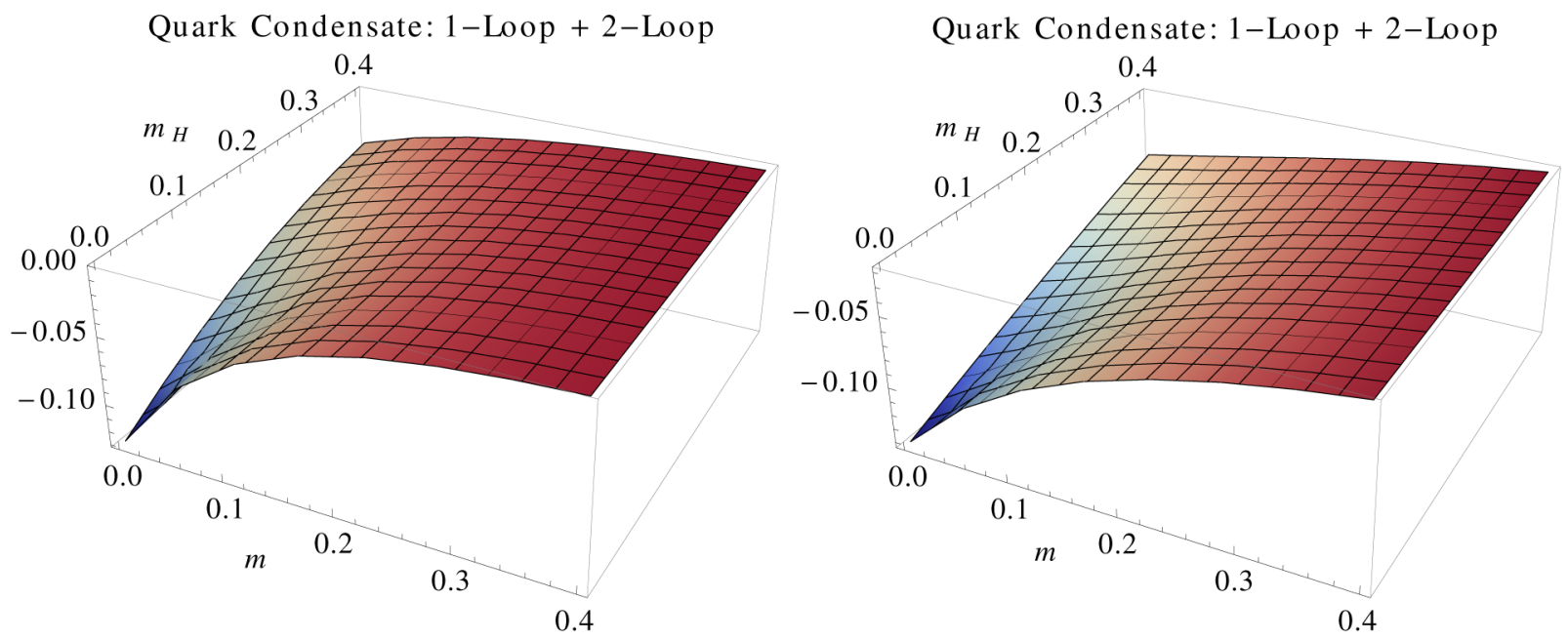

FIG. 3. Finite-temperature quark condensate: Sum of one- and two-loop contributions at $T=108 \mathrm{MeV}$ (left) and $T=215 \mathrm{MeV}$ (right).
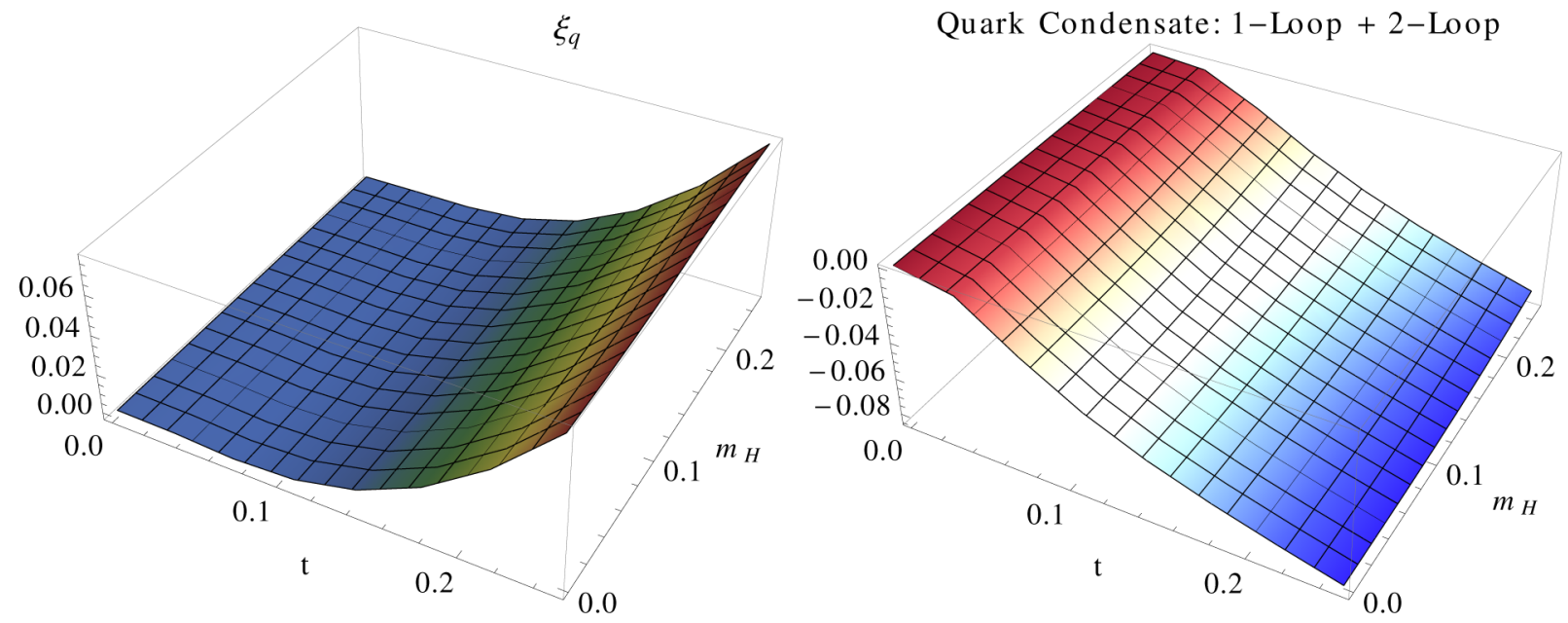

FIG. 4. Left: Magnitude and sign of the pion-pion interaction in the finite-temperature QCD quark condensate measured by $\xi_{q}\left(t, m, m_{H}\right)$ at the physical value $M_{\pi}=140 \mathrm{MeV}$. Right: Sum of one- and two-loop contributions of the finite-temperature quark condensate at the physical value $M_{\pi}$.

and corresponds to the charged pion mass in the chiral limit. The mass of the neutral pion, on the other hand, tends to zero in the chiral limit. ${ }^{9}$

We now address the question of how the quark condensate in the chiral limit behaves in weak magnetic fields. In this limit-implemented by $|q H| \ll T^{2}$-we have to expand the kinematical functions $h_{1}\left(M_{H}, T, 0\right)$ and $\tilde{h}_{1}\left(M_{H}, T, H\right)$ in Eq. (3.16) in the magnetic-fielddependent mass $M_{H}$, which leads to

\footnotetext{
${ }^{9}$ See Eqs. (2.16) and (2.17).
}

$$
\begin{aligned}
h_{1}\left(M_{H}, T, 0\right)= & h_{1}(0, T, 0)-\alpha \epsilon^{2} h_{2}(0, T, 0) \\
& +\frac{\alpha^{2} \epsilon^{4}}{2 !} h_{3}(0, T, 0)+\mathcal{O}\left(\epsilon^{2}\right), \\
\tilde{h}_{1}\left(M_{H}, T, H\right)= & \tilde{h}_{1}(0, T, H)-\alpha \epsilon^{2} \tilde{h}_{2}(0, T, H) \\
& +\frac{\alpha^{2} \epsilon^{4}}{2 !} \tilde{h}_{3}(0, T, H)+\mathcal{O}\left(\epsilon^{2}\right),
\end{aligned}
$$

with

$$
\alpha=\frac{\bar{l}_{6}-\bar{l}_{5}}{3} t^{2}, \quad t=\frac{T}{4 \pi F} .
$$


The structure of this infinite series of kinematical functions is analyzed in Appendix C. While the functions $h_{1}(0, T, 0)$ and $\tilde{h}_{1}(0, T, H)$ are well defined, it should be pointed out that for $r=2,3,4, \ldots$, the functions $h_{r}(0, T, 0)$ and $\tilde{h}_{r}(0, T, H)$ generate various types of divergences in the weak magnetic field expansion parameter $\epsilon$. The notation $h_{2}(0, T, 0), \quad \tilde{h}_{2}(0, T, H), \quad h_{3}(0, T, 0), \quad \tilde{h}_{3}(0, T, H), \ldots$ is therefore symbolic: it is understood that these functions contain inverse powers of $\epsilon$ as well as logarithms $\ln \epsilon$. These pieces-according to Eq. (3.18) - are then multiplied by even powers of $\epsilon$, in such a way that all divergences ultimately disappear in the quark condensate, as we show in Appendix C. In the expansion Eq. (3.18) we then keep all terms up to order $\epsilon^{2} \ln \epsilon$ but drop higher-order contributions as we indicate by $\mathcal{O}\left(\epsilon^{2}\right)$.

The outcome is the following series for the finitetemperature two-loop quark condensate in the chiral limit and in weak magnetic fields:

$$
\begin{aligned}
\frac{\langle\bar{q} q\rangle^{T}}{\langle 0|\bar{q} q| 0\rangle_{0}}= & -\frac{1}{8 F^{2}} T^{2}+\frac{1}{F^{2}}\left\{\frac{\left|I_{1 / 2}\right|}{8 \pi^{3 / 2}} \sqrt{\epsilon}-\frac{\ln 2}{16 \pi^{2}} \epsilon\right. \\
& \left.-\frac{\sqrt{2}-4}{8} \gamma \zeta\left(\frac{3}{2}\right) \epsilon^{3 / 2}+\frac{\gamma}{4 \pi} \epsilon^{2} \ln \epsilon+\mathcal{O}\left(\epsilon^{2}\right)\right\} T^{2} \\
& -\frac{1}{384 F^{4}} T^{4}+\frac{1}{F^{4}}\left\{\frac{\left|I_{1 / 2}\right|}{192 \pi^{3 / 2}} \sqrt{\epsilon}\right. \\
& \left.-\frac{\sqrt{2}-4}{192} \gamma \zeta\left(\frac{3}{2}\right) \epsilon^{3 / 2}+\frac{\gamma}{96 \pi} \epsilon^{2} \ln \epsilon+\mathcal{O}\left(\epsilon^{2}\right)\right\} T^{4} \\
& +\mathcal{O}\left(T^{6}\right) .
\end{aligned}
$$

Recall that $\epsilon$,

$$
\epsilon=\frac{|q H|}{T^{2}},
$$

is the relevant expansion parameter, while the other quantities are

$$
\begin{aligned}
I_{1 / 2} & =\int_{0}^{\infty} d \rho \rho^{-1 / 2}\left(\frac{1}{\sinh (\rho)}-\frac{1}{\rho}\right) \approx-1.516256, \\
\gamma & =\frac{\bar{l}_{6}-\bar{l}_{5}}{12 \pi} t^{2}, \quad t=\frac{T}{4 \pi F} .
\end{aligned}
$$

The first two lines of Eq. (3.20) refer to one-loop order $\left(\propto T^{2}\right)$, while the remaining two lines represent two-loop corrections $\left(\propto T^{4}\right)$. In the chiral limit, the series for the finite-temperature quark condensate in weak magnetic fields is thus characterized by square-root terms $\propto \sqrt{\epsilon}$, a term linear in $\epsilon$, followed by half-integer powers $\epsilon^{3 / 2}$ and logarithmic contributions of the form $\epsilon^{2} \ln \epsilon$. The remaining contributions involve even powers of $\epsilon$. Notice that the leading corrections-proportional to $\sqrt{\epsilon}$-come with a positive sign: in the chiral limit, as already illustrated by
Fig. 3, the finite-temperature quark condensate grows if the magnetic field is switched on.

The published results in Refs. [3-5,8-10] do not quite agree with the above representation. The correct series at one-loop order has been derived and discussed in Ref. [11]. The two-loop contribution in nonzero magnetic fields, displayed in the second brace of Eq. (3.20), again differs from the published two-loop result, Eq. (5.8) of Ref. [10]: the term

$$
\frac{5 \sqrt{|q H|} T^{3}}{1536 \pi F^{4}}
$$

in Eq. (5.8) of Ref. [10] should rather read

$$
\frac{\sqrt{|q H|} T^{3}}{192 \pi^{3 / 2} F^{4}}\left|I_{1 / 2}\right| \text {. }
$$

The numerical discrepancy is

$$
\frac{5}{1536 \pi} \approx 0.00103616, \quad \frac{\left|I_{1 / 2}\right|}{192 \pi^{3 / 2}} \approx 0.00141823 .
$$

Moreover, a term linear in $\epsilon$ in the second brace of Eq. (3.20) does not emerge in our expansioncontradicting the result announced in Ref. [10]. It should be emphasized that the series provided in the literature is restricted to linear order in $\epsilon$, while we have analyzed the full structure of the weak magnetic field expansion of the finite-temperature quark condensate in the chiral limit up to two loops.

\section{B. Zero-temperature quark condensate}

We now turn to the quark condensate at zero temperature:

$$
\langle 0|\bar{q} q| 0\rangle=-\frac{\langle 0|\bar{q} q| 0\rangle_{0}}{F^{2}} \frac{\mathrm{d} z_{0}}{\mathrm{~d} M^{2}} .
$$

Recall that $\langle 0|\bar{q} q| 0\rangle_{0}$ is the quark condensate at $T=0$, $H=0$ and $M=0$. On the basis of the representations Eqs. (2.21) and (2.23) for the vacuum energy density, we derive

$$
\begin{aligned}
& \frac{\langle 0|\bar{q} q| 0\rangle}{\langle 0|\bar{q} q| 0\rangle_{0}}=1-\frac{\bar{l}_{3}-4 \bar{h}_{1}}{32 \pi^{2}} \frac{M^{2}}{F^{2}}-\frac{K_{1}}{F^{2}}+\frac{3 \bar{l}_{3}}{1024 \pi^{4}} \frac{M^{4}}{F^{4}} \\
& -\frac{9 \bar{l}_{3}\left(\bar{c}_{10}+2 \bar{c}_{11}\right)}{1024 \pi^{4}} \frac{M^{4}}{F^{4}}-\frac{\bar{l}_{6}-\bar{l}_{5}}{768 \pi^{4}} \frac{|q H|^{2}}{F^{4}} \\
& +\frac{\left(\bar{l}_{6}-\bar{l}_{5}\right) \bar{c}_{34}}{768 \pi^{4}} \frac{|q H|^{2}}{F^{4}}-\frac{1}{32 \pi^{2}} \frac{M^{2}}{F^{4}} K_{1} \\
& +\frac{\bar{l}_{3}}{16 \pi^{2}} \frac{M^{2}}{F^{4}} K_{1}+\frac{\bar{l}_{3}}{32 \pi^{2}} \frac{M^{4}}{F^{4}} \frac{\mathrm{d} K_{1}}{\mathrm{~d} M^{2}} \\
& -\frac{\left(\bar{l}_{6}-\bar{l}_{5}\right)}{48 \pi^{2}} \frac{|q H|^{2}}{F^{4}} \frac{\mathrm{d} K_{1}}{\mathrm{~d} M^{2}} \text {. }
\end{aligned}
$$


The explicit expressions for $K_{1}$ and $\mathrm{d} K_{1} / \mathrm{d} M^{2}$,

$$
\begin{aligned}
K_{1}= & \frac{M^{2}}{16 \pi^{2}}-\frac{M^{2}}{16 \pi^{2}} \ln \frac{M^{2}}{2|q H|}+\frac{|q H|}{8 \pi^{2}} \ln \Gamma\left(\frac{M^{2}}{2|q H|}+\frac{1}{2}\right) \\
& -\frac{|q H|}{16 \pi^{2}} \ln 2 \pi, \\
\frac{\mathrm{d} K_{1}}{\mathrm{~d} M^{2}}= & \frac{1}{16 \pi^{2}} \ln \frac{|q H|}{M^{2}}+\frac{1}{16 \pi^{2}} \Psi\left(\frac{M^{2}}{2|q H|}+\frac{1}{2}\right)+\frac{\ln 2}{16 \pi^{2}},
\end{aligned}
$$

are derived in Appendix B. The series for the quark condensate is organized according to ascending powers of $M^{2}$ and $|q H|$-both quantities count as order $p^{2}$. The respective coefficients depend in a nontrivial manner on the ratio $M^{2} /|q H|$ and involve renormalized NLO and NNLO effective constants. Let us compare our result with the literature.

The focus of the two-loop CHPT calculation presented in Ref. [7] was to determine the shift in the zero-temperature quark condensate caused by an external (electro)magnetic field. Our expression, Eq. (3.27), is consistent with the twoloop vacuum energy density derived in Eq. (21) of this reference but goes beyond it, since we have derived the whole two-loop representation for the quark condensatenot just the terms induced by the magnetic field.

To analyze the chiral limit of the zero-temperature quark condensate in finite magnetic fields, we invoke the behavior of the NLO and NNLO effective constants. According to Appendix A we have

$$
\begin{aligned}
\bar{l}_{3}, \bar{l}_{5}, \bar{l}_{6} & \propto \ln M^{2}, \\
\bar{c}_{34} & \propto \ln M^{2}, \\
\bar{c}_{10}+2 \bar{c}_{11} & \propto \ln M^{2},
\end{aligned}
$$

i.e., the renormalized NLO and NNLO effective constants explode in the limit $M \rightarrow 0$. But note that in the quark condensate these constants are multiplied by powers of $M^{2}$ such that the chiral limit is in fact unproblematic. While some terms in Eq. (3.27) hence disappear in the chiral limit, only the following two terms:

$$
\frac{\left(\bar{l}_{6}-\bar{l}_{5}\right)}{768 \pi^{4}} \bar{c}_{34} \frac{|q H|^{2}}{F^{4}}-\frac{\left(\bar{l}_{6}-\bar{l}_{5}\right)}{768 \pi^{4}} \frac{|q H|^{2}}{F^{4}} \ln \left(\frac{|q H|}{M^{2}}\right)
$$

need special consideration, as they both explode in the chiral limit. However, writing the NNLO effective constant $\bar{c}_{34}$ as

$$
\bar{c}_{34}=\ln \left(\frac{\Lambda_{34}^{2}}{M_{\pi}^{2}}\right)
$$

where $\Lambda_{34}$ is the renormalization group invariant scale associated with $\bar{c}_{34}$, the two terms can be merged such that the zero-temperature quark condensate in nonzero magnetic fields is well defined in the chiral limit, taking the form

$$
\begin{aligned}
\frac{\langle 0|\bar{q} q| 0\rangle}{\langle 0|\bar{q} q| 0\rangle_{0}}= & 1+\frac{\ln 2}{16 \pi^{2}} \frac{|q H|}{F^{2}}-\frac{\left(\bar{l}_{6}-\bar{l}_{5}\right)}{768 \pi^{4}} \frac{|q H|^{2}}{F^{4}} \ln \left(\frac{|q H|}{\Lambda_{34}^{2}}\right) \\
& -\frac{\left(\bar{l}_{6}-\bar{l}_{5}\right)}{768 \pi^{4}} \frac{|q H|^{2}}{F^{4}}-\frac{\left(\bar{l}_{6}-\bar{l}_{5}\right)}{768 \pi^{4}} \frac{|q H|^{2}}{F^{4}}\left(\frac{\Gamma^{\prime}\left(\frac{1}{2}\right)}{\Gamma\left(\frac{1}{2}\right)}+\ln 2\right) .
\end{aligned}
$$

Notice that the $\ln M^{2}$ dependence in the combination $\bar{l}_{6}-\bar{l}_{5}$ cancels, and we can write

$$
\bar{l}_{6}-\bar{l}_{5}=\ln \left(\frac{\Lambda_{6}^{2}}{\Lambda_{5}^{2}}\right),
$$

where $\Lambda_{5}$ and $\Lambda_{6}$ are the respective renormalization group invariant scales associated with the NLO effective constants $\bar{l}_{5}$ and $\bar{l}_{6}$.

\section{CONCLUSIONS}

We have explored the behavior of the quark condensate subjected to an external magnetic field within the framework of chiral perturbation theory. Unlike previous two-loop evaluations by other authors, we have used a coordinate-space representation.

Regarding the finite-temperature quark condensate in the chiral limit and in weak magnetic fields, we have pointed out various errors that have occurred in the literature and have provided the correct series. At order $T^{2}$-and in terms of the expansion parameter $\epsilon=|q H| / T^{2}$ - the leading contribution is proportional to $\sqrt{\epsilon}$, followed by a term linear in $\epsilon$, a half-integer power $\epsilon^{3 / 2}$ and a logarithmic contribution $\epsilon^{2} \ln \epsilon$. The remaining contributions involve even powers of $\epsilon$. At order $T^{4}$ the pattern repeats itself with the exception that a term linear in $\epsilon$ does not occur.

Leaving the weak magnetic field limit, we have investigated the impact of the magnetic field on the quark condensate at finite temperature. Emphasis was put on the effect of the pion-pion interaction which constitutes up to about $10 \%$ for arbitrary pion masses but also in the real world where $M_{\pi}=140 \mathrm{MeV}$. The interaction is largest in the chiral limit. The finite-temperature quark condensate (sum of one- and two-loop contributions) at fixed temperature and fixed pion mass grows monotonically when the magnetic field strength increases. Again, the effect is most pronounced in the chiral limit.

Finally we have derived the two-loop representation for the QCD vacuum energy density and the quark condensate at zero temperature. We have complemented earlier studies by other authors, by providing the full two-loop representation, i.e., not just the terms that emerge on account of the nonzero magnetic field. 
A natural-but highly nontrivial-step is to extend the present analysis to the three-loop level, in analogy to the three-loop analysis in zero magnetic field given in the pioneering article [44], based on a coordinate-space representation of CHPT. Corresponding work is in progress.

\section{ACKNOWLEDGMENTS}

The author gratefully acknowledges $\mathrm{H}$. Leutwyler and J. Bijnens for correspondence. Special thanks to J. Bijnens for sharing unpublished results on the order- $p^{6}$ zerotemperature quark condensate.

\section{APPENDIX A: ORDER- $p^{6}$ FREE ENERGY DENSITY AT $T=0$}

\section{Low-energy effective constants at NLO and NNLO}

The aim of the present Appendix is to discuss the renormalization group running of the NLO and NNLO effective constants $l_{i}^{r}$ and $c_{i}^{r}$ in some detail and then to provide a definition of the renormalized NNLO effective constants $\bar{c}_{i}$-in analogy to the definition of the renormalized NLO quantities $\bar{l}_{i}$.

The NNLO effective constants $c_{i}$ that appear in $\mathcal{L}_{\text {eff }}^{6}$ are defined in Ref. [41] as

$$
c_{i}=\frac{(c \mu)^{2(d-4)}}{F^{2}}\left\{c_{i}^{r}-\gamma_{i}^{(2)} \Lambda^{2}-\gamma_{i}^{(1)} \Lambda-\gamma_{i}^{(L)} \Lambda\right\},
$$

with

$$
\Lambda=\frac{1}{16 \pi^{2}} \frac{1}{d-4}, \quad \ln c=-\frac{1}{2}\left[\ln 4 \pi+\Gamma^{\prime}(1)+1\right] .
$$

The quantities $\gamma_{i}^{(1)}$ and $\gamma_{i}^{(2)}$ are pure numbers and the $c_{i}^{r}$ are the renormalized running NNLO effective constants. For the definition of the NLO effective constants $l_{i}$ and $h_{i}$ that appear in $\mathcal{L}_{\text {eff }}^{4}$, on the other hand, we adopt the definition given in the original Ref. [42]:

$$
\begin{aligned}
l_{i} & =l_{i}^{r}+\gamma_{i} \lambda, \\
h_{i} & =h_{i}^{r}+\delta_{i} \lambda,
\end{aligned}
$$

where

$$
\begin{aligned}
\lambda & =\frac{1}{2}(4 \pi)^{-d / 2} \Gamma\left(1-\frac{1}{2} d\right) \mu^{d-4} \\
& =\frac{\mu^{d-4}}{16 \pi^{2}}\left[\frac{1}{d-4}-\frac{1}{2}\left\{\ln 4 \pi+\Gamma^{\prime}(1)+1\right\}+\mathcal{O}(d-4)\right] .
\end{aligned}
$$

The $\gamma_{i}$ are pure numbers and the $l_{i}^{r}$ are the renormalized running NLO effective constants. The definition [Eq. (A3)] can be rewritten as

$$
l_{i}=l_{i}^{r}+(c \mu)^{d-4} \gamma_{i} \Lambda .
$$

Note that the $\gamma_{i}$ also show up in $\gamma_{i}^{(L)}$ [Eq. (A1)] in the form of

$$
\gamma_{i}^{(L)}=\sum_{j} \gamma_{i j}^{(L)}(c \mu)^{-(d-4)} l_{j}^{r}
$$

where the coefficients $\gamma_{i j}^{(L)}$ are again pure numbers.

Since the $c_{i}$ do not depend on the renormalization scale $\mu$, one concludes that the renormalization group running of the NNLO effective constants $c_{i}^{r}$ is

$$
\mu \frac{\mathrm{d} c_{i}^{r}}{\mathrm{~d} \mu}=-2(d-4) c_{i}^{r}+\frac{\gamma_{i}^{(1)}}{8 \pi^{2}}+\frac{\gamma_{i}^{(L)}}{16 \pi^{2}} .
$$

In the above derivation we have used the fact that the NLO effective constants $l_{i}^{r}$ themselves obey the running

$$
\mu \frac{\mathrm{d} l_{i}^{r}}{\mathrm{~d} \mu}=-\frac{\gamma_{i}}{16 \pi^{2}}(c \mu)^{d-4},
$$

which follows from the fact that the $l_{i}$ do not depend on $\mu$. Furthermore, with the Weinberg consistency condition [45]

$$
-2 \gamma_{i}^{(2)}+\sum_{j} \gamma_{i j}^{(L)} \gamma_{j}=0,
$$

a divergence linear in $\Lambda$ has been eliminated in Eq. (A7).

Instead of the NLO quantities $l_{i}^{r}$ that depend on the renormalization scale, alternatively one often uses the NLO effective constants $\bar{l}_{i}$ that are $\mu$ independent. The connection between the two is [42]

$$
l_{i}^{r}=\frac{\gamma_{i}}{32 \pi^{2}}\left(\bar{l}_{i}+\ln \frac{M^{2}}{\mu^{2}}\right) .
$$

Let us transfer this connection to NNLO. The specific NNLO effective constants that appear in the vacuum energy density are $c_{10}, c_{11}$ and $c_{34}$, where the last one only matters when a magnetic field is present. Following Ref. [41]—but using the convention (A3) - it reads

$$
c_{34}=\frac{(c \mu)^{2(d-4)}}{F^{2}} c_{34}^{r}+\frac{l_{5}^{r}-\frac{1}{2} l_{6}^{r}}{F^{2}} \lambda .
$$

Explicitly, the running of $c_{34}^{r}$ is given by

$$
\frac{\mathrm{d} c_{34}^{r}}{\mathrm{~d} \mu^{2}}=-\frac{l_{5}^{r}-\frac{1}{2} l_{6}^{r}}{32 \pi^{2} \mu^{2}} .
$$

In analogy to the above definition for the NLO constants $\bar{l}_{i}$ [Eq. (A10)] that is based on the running (A8), we define the renormalized NNLO effective constant $\bar{c}_{34}$ as 


$$
c_{34}^{r}=\frac{\bar{l}_{6}-\bar{l}_{5}}{6144 \pi^{4}} \bar{c}_{34}+\frac{\bar{l}_{6}-\bar{l}_{5}}{6144 \pi^{4}} \ln \frac{M^{2}}{\mu^{2}} .
$$

Note that we have used

$$
\gamma_{5}=-\frac{1}{6}, \quad \gamma_{6}=-\frac{1}{3}
$$

Since $c_{34}^{r}$ does not depend on $M$, we conclude

$$
\frac{\mathrm{d} \bar{c}_{34}}{\mathrm{~d} M^{2}}=-\frac{1}{M^{2}} .
$$

The NNLO constant $\bar{c}_{34}$ hence obeys the same simple relation as the NLO constants $\bar{l}_{i}$ :

$$
\frac{\mathrm{d} \bar{l}_{i}}{\mathrm{~d} M^{2}}=-\frac{1}{M^{2}}
$$

Next we consider the NNLO effective constants $c_{10}$ and $c_{11}$ that arise in the tree-level contribution $z_{6 C}$ in the absence of the magnetic field. They are defined as (see Ref. [41])

$$
\begin{aligned}
& c_{10}=\frac{(c \mu)^{2(d-4)}}{F^{2}} c_{10}^{r}+\frac{3}{64 F^{2}} \lambda^{2}-\frac{1}{F^{2}}\left(\frac{3}{16} l_{3}^{r}+\frac{1}{16} l_{7}^{r}\right) \lambda, \\
& c_{11}=\frac{(c \mu)^{2(d-4)}}{F^{2}} c_{11}^{r}-\frac{9}{128 F^{2}} \lambda^{2}+\frac{1}{F^{2}}\left(\frac{9}{32} l_{3}^{r}+\frac{1}{32} l_{7}^{r}\right) \lambda .
\end{aligned}
$$

Note that in the linear combination $c_{10}+2 c_{11}$-as it appears in the vacuum energy density at order $p^{6}$-the dependence on $l_{7}^{r}$ cancels and we are left with

$$
c_{10}+2 c_{11}=\frac{(c \mu)^{2(d-4)}}{F^{2}}\left(c_{10}^{r}+2 c_{11}^{r}\right)-\frac{3}{32 F^{2}} \lambda^{2}+\frac{3}{8 F^{2}} l_{3}^{r} \lambda .
$$

Since the $c_{i}$ do not depend on the renormalization scale $\mu$, we conclude

$$
\frac{\mathrm{d}\left(c_{10}^{r}+2 c_{11}^{r}\right)}{\mathrm{d} \mu^{2}}=-\frac{3 l_{3}^{r}}{256 \pi^{2}} \frac{1}{\mu^{2}} .
$$

Equivalently, by making the replacement $l_{3}^{r} \rightarrow \bar{l}_{3}$,

$$
l_{3}^{r}=\frac{\gamma_{3}}{32 \pi^{2}}\left(\bar{l}_{3}+\ln \frac{M^{2}}{\mu^{2}}\right), \quad \gamma_{3}=-\frac{1}{2},
$$

we can write

$\frac{\mathrm{d}\left(c_{10}^{r}+2 c_{11}^{r}\right)}{\mathrm{d} \mu^{2}}=\frac{3 \bar{l}_{3}}{16384 \pi^{4}} \frac{1}{\mu^{2}}+\frac{3}{16384 \pi^{4}} \frac{1}{\mu^{2}} \ln \frac{M^{2}}{\mu^{2}}$.
This leads us to the definition of the renormalized combination $\bar{c}_{10}+2 \bar{c}_{11}$ as

$$
\begin{aligned}
c_{10}^{r}+2 c_{11}^{r}= & -\frac{3 \bar{l}_{3}}{16384 \pi^{4}}\left(\bar{c}_{10}+2 \bar{c}_{11}\right)-\frac{3 \bar{l}_{3}}{16384 \pi^{4}} \ln \frac{M^{2}}{\mu^{2}} \\
& -\frac{3}{32768 \pi^{4}}\left(\ln \frac{M^{2}}{\mu^{2}}\right)^{2} .
\end{aligned}
$$

By construction, the linear combination $\bar{c}_{10}+2 \bar{c}_{11}$ is independent of $\mu$, much like $\bar{c}_{34}$ and the $\bar{l}_{i}$. Because the expression $c_{10}^{r}+2 c_{11}^{r}$ does not depend on $M$, we also conclude

$$
\frac{\mathrm{d}\left(\bar{c}_{10}+2 \bar{c}_{11}\right)}{\mathrm{d} M^{2}}=-\frac{1}{M^{2}}+\frac{1}{M^{2}} \frac{\bar{c}_{10}+2 \bar{c}_{11}}{\bar{l}_{3}} .
$$

\section{Isolating UV divergences}

Here we focus on the zero-temperature contributions in the free energy density that emerge at order $p^{6}$ due to the three diagrams $6 A-C$ displayed Fig. 1. The unrenormalized expressions that contain both $T=0$ and finite-temperature pieces are

$$
\begin{aligned}
& z_{6 A}=\frac{M^{2}}{2 F^{2}} G_{1}^{ \pm} G_{1}^{0}-\frac{M^{2}}{8 F^{2}} G_{1}^{0} G_{1}^{0}, \\
& z_{6 B}=\left(4 l_{5}-2 l_{6}\right) \frac{|q H|^{2}}{F^{2}} G_{1}^{ \pm}+2 l_{3} \frac{M^{4}}{F^{2}} G_{1}^{ \pm}+l_{3} \frac{M^{4}}{F^{2}} G_{1}^{0}, \\
& z_{6 C}=-16\left(c_{10}+2 c_{11}\right) M^{6}-8 c_{34}|q H|^{2} M^{2},
\end{aligned}
$$

where $G_{1}^{ \pm}$and $G_{1}^{0}$ are the thermal pion propagators evaluated at the coordinate origin $x=0$ :

$$
G_{1}^{ \pm}=G^{ \pm}(0), \quad G_{1}^{0}=G^{0}(0) .
$$

Inserting the decomposition of thermal propagators into zero-temperature and finite-temperature pieces [defined in Eq. (2.14)]

$$
\begin{aligned}
G_{1}^{ \pm} & =\Delta^{ \pm}(0)+\tilde{g}_{1}(M, T, H)+g_{1}(M, T, 0), \\
G_{1}^{0} & =\Delta^{0}(0)+g_{1}(M, T, 0),
\end{aligned}
$$

and using the representations of the zero-temperature propagators $\Delta^{ \pm}(0)$ and $\Delta^{0}(0)$,

$$
\Delta^{ \pm}(0)=2 M^{2} \lambda+K_{1}, \quad \Delta^{0}(0)=2 M^{2} \lambda,
$$

with $K_{1}$ and $\lambda$ as 


$$
\begin{aligned}
K_{1}= & \frac{|q H|^{(d / 2)-1}}{(4 \pi)^{d / 2}} \int_{0}^{\infty} \mathrm{d} \rho \rho^{-(d / 2)+1} \\
& \times \exp \left(-\frac{M^{2}}{|q H|} \rho\right)\left(\frac{1}{\sinh (\rho)}-\frac{1}{\rho}\right), \\
\lambda= & \frac{1}{2}(4 \pi)^{-d / 2} \Gamma\left(1-\frac{1}{2} d\right) M^{d-4} \\
= & \frac{M^{d-4}}{16 \pi^{2}}\left[\frac{1}{d-4}-\frac{1}{2}\left\{\ln 4 \pi+\Gamma^{\prime}(1)+1\right\}+\mathcal{O}(d-4)\right],
\end{aligned}
$$

we obtain

$$
\begin{aligned}
z_{6 A}^{0}= & \frac{3 M^{6}}{2 F^{2}} \lambda^{2}+\frac{M^{4}}{F^{2}} K_{1} \lambda, \\
z_{6 B}^{0}= & 6 l_{3} \frac{M^{6}}{F^{2}} \lambda+2 l_{3} \frac{M^{4}}{F^{2}} K_{1}+\left(8 l_{5}-4 l_{6}\right) \frac{M^{2}|q H|^{2}}{F^{2}} \lambda \\
& +\left(4 l_{5}-2 l_{6}\right) \frac{|q H|^{2}}{F^{2}} K_{1}, \\
z_{6 C}^{0}= & -16\left(c_{10}+2 c_{11}\right) M^{6}-8 c_{34}|q H|^{2} M^{2} .
\end{aligned}
$$

The upper index " 0 " signals that we are considering the $T=0$ part only. ${ }^{10}$ To isolate the UV divergences in this unrenormalized expression, we use the conventions for the NLO and NNLO effective constants $l_{i}$ and $c_{i}$, respectively, that we have provided in Appendix A 1. One finds that in the sum of the three diagrams, all UV divergences disappear and the renormalized order- $p^{6}$ vacuum energy density takes the form

$$
\begin{aligned}
z_{0}^{[6]}= & z_{6 A}^{0}+z_{6 B}^{0}+z_{6 C}^{0} \\
= & \frac{3 \bar{l}_{3}\left(\bar{c}_{10}+2 \bar{c}_{11}\right)}{1024 \pi^{4}} \frac{M^{6}}{F^{2}}-\frac{\left(\bar{l}_{6}-\bar{l}_{5}\right) \bar{c}_{34}}{768 \pi^{4}} \frac{|q H|^{2} M^{2}}{F^{2}} \\
& -\frac{\bar{l}_{3}}{32 \pi^{2}} \frac{M^{4}}{F^{2}} K_{1}+\frac{\left(\bar{l}_{6}-\bar{l}_{5}\right)}{48 \pi^{2}} \frac{|q H|^{2}}{F^{2}} K_{1} .
\end{aligned}
$$

The above representation is renormalization-scale independent. This constitutes a nontrivial check of our calculation.

The cancellation of divergences demonstrates that the renormalization of NLO and NNLO effective constants also perfectly works in presence of a homogeneous external magnetic field and that the effective field theory method is fully consistent.

In particular, charge renormalization is not an issue in the present calculation of the vacuum energy density where photons are described in terms of an external classical field. Quantum fluctuations of the photon field are not relevant here; only fluctuations of the pion field are important. The quantization of the electromagnetic field would indeed lead to corrections proportional to the fine structure constant,

\footnotetext{
${ }^{10}$ The finite-temperature contribution $z^{T}$ is given by Eq. (2.13).
}

but the respective diagrams would contain internal photon lines which are not relevant to the free energy density.

\section{APPENDIX B: ANALYSIS OF THE INTEGRAL $K_{1}$}

To analyze the free energy density and the quark condensate in the chiral limit, we must have a closer look at the dimensionally regularized integral $K_{1}$ :

$$
\begin{aligned}
K_{1}= & \frac{|q H|^{(d / 2)-1}}{(4 \pi)^{d / 2}} \int_{0}^{\infty} \mathrm{d} \rho \rho^{-(d / 2)+1} \\
& \times \exp \left(-\frac{M^{2}}{|q H|} \rho\right)\left(\frac{1}{\sinh (\rho)}-\frac{1}{\rho}\right) .
\end{aligned}
$$

To this end we first consider the integral $I_{2}$, defined in (A1) of Ref. [11] as

$$
I_{2}=-\frac{|q H|^{d / 2}}{(4 \pi)^{d / 2}} \int_{0}^{\infty} d \rho \rho^{-d / 2}\left(\frac{1}{\sinh (\rho)}-\frac{1}{\rho}\right) \exp \left(-\frac{M^{2}}{|q H|} \rho\right) .
$$

Comparing these representations, one concludes

$$
\begin{aligned}
K_{1} & =\frac{\mathrm{d} I_{2}}{\mathrm{~d} M^{2}}, \\
\frac{\mathrm{d} K_{1}}{\mathrm{~d} M^{2}} & =\frac{\mathrm{d}^{2} I_{2}}{\left(\mathrm{~d} M^{2}\right)^{2}} .
\end{aligned}
$$

Using the property of the Riemann zeta function

$$
\lim _{s \rightarrow 1} \zeta(s, q)=\frac{1}{s-1}-\frac{\Gamma^{\prime}(q)}{\Gamma(q)}
$$

where

$$
\zeta(s, q)=\sum_{n=0}^{\infty} \frac{1}{(q+n)^{s}},
$$

the second relation in Eq. (B3) yields ${ }^{11}$

$$
\frac{\mathrm{d} K_{1}}{\mathrm{~d} M^{2}}=\frac{1}{16 \pi^{2}} \ln \frac{|q H|}{M^{2}}+\frac{1}{16 \pi^{2}} \Psi\left(\frac{M^{2}}{2|q H|}+\frac{1}{2}\right)+\frac{\ln 2}{16 \pi^{2}},
$$

where $\Psi(x)$ is the polygamma function

$$
\Psi(x)=\frac{\Gamma^{\prime}(x)}{\Gamma(x)} .
$$

\footnotetext{
${ }^{11}$ The physical limit $d \rightarrow 4$ is straightforward and does not pose any problems.
} 
The expression for $K_{1}$ is obtained by integration:

$$
\begin{aligned}
K_{1}= & \frac{M^{2}}{16 \pi^{2}}-\frac{M^{2}}{16 \pi^{2}} \ln \frac{M^{2}}{2|q H|}+\frac{|q H|}{8 \pi^{2}} \ln \Gamma\left(\frac{M^{2}}{2|q H|}+\frac{1}{2}\right) \\
& +C(|q H|) .
\end{aligned}
$$

The integration constant $C(|q H|)$ can be determined by setting $M=0$ in the equation above and in the original representation, Eq. (B1). One identifies

$$
C(|q H|)=-\frac{|q H|}{16 \pi^{2}} \ln 2 \pi .
$$

While $K_{1}$ appears in the free energy density, the derivative $\mathrm{d} K_{1} / \mathrm{d} M^{2}$ is relevant in the quark condensate.

\section{APPENDIX C: BOSE FUNCTIONS IN THE CHIRAL LIMIT}

The finite-temperature representation of the quark condensate in the chiral limit [Eq. (3.16)] features an infinite series of kinematical Bose functions $g_{r}$ and $\tilde{g}_{r}$ that has to be resummed because of the weak magnetic field expansion Eq. (3.18). This is the main focus of the present Appendix. The aim is to provide explicit expressions up to order $\epsilon^{2} \ln \epsilon$ in the finite-temperature quark condensate.

We first consider the second type of functions ${ }^{12}$

$$
\begin{aligned}
\tilde{g}_{r}\left(M_{\pi}^{ \pm}, T, H\right)= & \frac{\epsilon}{(4 \pi)^{r+1}} T^{d-2 r} \int_{0}^{\infty} \mathrm{d} \rho \rho^{-(d / 2)+r} \\
& \times \exp \left(\frac{-\left(M_{\pi}^{ \pm}\right)^{2}}{4 \pi T^{2}} \rho\right)\left(\frac{1}{\sinh (\epsilon \rho / 4 \pi)}-\frac{4 \pi}{\epsilon \rho}\right) \\
& \times\left[S\left(\frac{1}{\rho}\right)-1\right] .
\end{aligned}
$$

The crucial point is that-in the chiral limit-the mass $M_{\pi}^{ \pm}$ of the charged pions does not tend to zero. Rather, according to Eq. (2.16), a magnetic-field-dependent mass term survives the chiral limit:

$$
M_{H}^{2}=\frac{\bar{l}_{6}-\bar{l}_{5}}{48 \pi^{2}} \frac{|q H|^{2}}{F^{2}}=\frac{16 \pi^{2}}{3}\left(\bar{l}_{6}-\bar{l}_{5}\right) t^{4} F^{2} \epsilon^{2},
$$

with

$$
t=\frac{T}{4 \pi F}
$$

The pertinent expansion parameter in the weak magnetic field limit $|q H| \ll T^{2}$ is

$$
\epsilon=\frac{|q H|}{T^{2}} .
$$

To isolate divergences in the kinematical functions $\tilde{g}_{r}$ (where $r=0,1,2, \ldots)$ that arise in the limit $\epsilon \rightarrow 0$ ( $T$ held fixed while $H \rightarrow 0)$, we decompose $\tilde{g}_{r}\left(M_{H}, T, H\right)$ into two pieces:

$$
\begin{aligned}
\tilde{g}_{r}\left(M_{H}, T, H\right)= & \frac{\epsilon T^{d-2 r}}{(4 \pi)^{r+1}} \int_{0}^{1} \mathrm{~d} \rho \rho^{-(d / 2)+r} e^{-\gamma \epsilon^{2} \rho} \\
& \times\left(\frac{1}{\sinh (\epsilon \rho / 4 \pi)}-\frac{4 \pi}{\epsilon \rho}\right)\left[S\left(\frac{1}{\rho}\right)-1\right] \\
& +\frac{\epsilon T^{d-2 r}}{(4 \pi)^{r+1}} \int_{1}^{\infty} \mathrm{d} \rho \rho^{-(d / 2)+r} e^{-\gamma \epsilon^{2} \rho} \\
& \times\left(\frac{1}{\sinh (\epsilon \rho / 4 \pi)}-\frac{4 \pi}{\epsilon \rho}\right)\left[S\left(\frac{1}{\rho}\right)-1\right] \\
= & I_{a}+I_{b},
\end{aligned}
$$

where

$$
\gamma=\frac{\bar{l}_{6}-\bar{l}_{5}}{12 \pi} t^{2}
$$

The first integral $I_{a}$ exists for integer $r=0,1,2, \ldots$. Taylor expanding the integrand in the parameter $\epsilon$, we obtain a series with ascending even powers of $\epsilon$ for $r=0,1,2, \ldots$ :

$$
\alpha_{1} \epsilon^{2}+\alpha_{2} \epsilon^{4}+\alpha_{3} \epsilon^{6}+\mathcal{O}\left(\epsilon^{8}\right) .
$$

The explicit coefficients are irrelevant for our purposes because the respective terms do not contribute to the quark condensate at the accuracy we are interested in (up to $\epsilon^{2} \ln \epsilon$ in the finite-temperature quark condensate). In particular, no $\epsilon$ divergences come from here.

We thus examine the second integral $I_{b}$ in Eq. (C5) that we process by using the Jacobi identity

$$
S\left(\frac{1}{z}\right)=\sqrt{z} S(z)
$$

We then obtain the three integrals

$$
\begin{aligned}
I_{b}= & \frac{\epsilon T^{d-2 r}}{(4 \pi)^{r+1}} \int_{1}^{\infty} \mathrm{d} \rho \rho^{r-(d / 2)+(1 / 2)} e^{-\gamma \epsilon^{2} \rho}\left(\frac{1}{\sinh (\epsilon \rho / 4 \pi)}-\frac{4 \pi}{\epsilon \rho}\right)[S(\rho)-1] \\
& +\frac{\epsilon T^{d-2 r}}{(4 \pi)^{r+1}} \int_{1}^{\infty} \mathrm{d} \rho \rho^{r-(d / 2)+(1 / 2)} e^{-\gamma \epsilon^{2} \rho}\left(\frac{1}{\sinh (\epsilon \rho / 4 \pi)}-\frac{4 \pi}{\epsilon \rho}\right)-\frac{\epsilon T^{d-2 r}}{(4 \pi)^{r+1}} \int_{1}^{\infty} \mathrm{d} \rho \rho^{r-(d / 2)} e^{-\gamma \epsilon^{2} \rho}\left(\frac{1}{\sinh (\epsilon \rho / 4 \pi)}-\frac{4 \pi}{\epsilon \rho}\right) \\
= & I_{b 1}+I_{b 2}+I_{b 3} .
\end{aligned}
$$

\footnotetext{
${ }^{12}$ It should be noted that the functions $\tilde{g}_{r}$ and $g_{r}$ - up to temperature powers—coincide with the functions $\tilde{h}_{r}$ and $h_{r}$. The conversion is given by Eq. (3.8).
} 
The first one- $I_{b 1}$-exists for integer $r=0,1,2, \ldots$. Taylor expanding the integrand and then integrating term by term we get a series of the form

$$
\beta_{1} \epsilon^{2}+\beta_{2} \epsilon^{4}+\beta_{3} \epsilon^{6}+\mathcal{O}\left(\epsilon^{8}\right) .
$$

Again, the coefficients are irrelevant at the accuracy we are interested in. To isolate potential $\epsilon$ divergences in $I_{b 2}$, we write the integration limits as

$$
\begin{aligned}
I_{b 2}= & \frac{\epsilon T^{d-2 r}}{(4 \pi)^{r+1}} \int_{0}^{\infty} \mathrm{d} \rho \rho^{r-(d / 2)+(1 / 2)} e^{-\gamma \epsilon^{2} \rho} \\
& \times\left(\frac{1}{\sinh (\epsilon \rho / 4 \pi)}-\frac{4 \pi}{\epsilon \rho}\right) \\
& -\frac{\epsilon T^{d-2 r}}{(4 \pi)^{r+1}} \int_{0}^{1} \mathrm{~d} \rho \rho^{r-(d / 2)+(1 / 2)} e^{-\gamma \epsilon^{2} \rho} \\
& \times\left(\frac{1}{\sinh (\epsilon \rho / 4 \pi)}-\frac{4 \pi}{\epsilon \rho}\right) .
\end{aligned}
$$

The first expression can be integrated analytically:

$$
\begin{aligned}
I_{b 2}^{[1]}= & T^{d-2 r} \Gamma\left(r-\frac{3}{2}\right)\left[-\frac{\gamma^{(3 / 2)-r}}{(4 \pi)^{r}} \epsilon^{3-2 r}\right. \\
& \left.+2^{-r-(5 / 2)} \pi^{-3 / 2}(2 r-3) \zeta\left(r-\frac{1}{2}, \frac{1}{2}+2 \pi \gamma \epsilon\right) \epsilon^{(3 / 2)-r}\right],
\end{aligned}
$$

where the generalized Riemann zeta function is defined as

$$
\zeta(s, a)=\sum_{k=0}^{\infty} \frac{1}{(k+a)^{s}} .
$$

One notices that the integral $I_{b 2}^{[1]}$ (for integer $r \geq 2$ ) leads to $\epsilon$ divergences in the functions $\tilde{g}_{r}$, namely

$$
\tilde{g}_{r} \propto \frac{1}{\epsilon^{2 r-3}}, \frac{1}{\epsilon^{r-(3 / 2)}} .
$$

As it turns out, these are indeed the leading divergences in the Bose functions $\tilde{g}_{r}$. With the second expression- $I_{b 2}^{[2]}-$ in Eq. (C11) we proceed as before: Taylor expanding the integrand again gives rise to a series displaying even $\epsilon$ powers whose respective coefficients are of no concern to us.

Finally, we analyze the remaining third integral $I_{b 3}$ in Eq. (C9). Regularizing it with $N \gg 1$,

$$
\begin{aligned}
I_{b 3}= & \lim _{N \rightarrow \infty}-\frac{\epsilon T^{d-2 r}}{(4 \pi)^{r+1}} \int_{1}^{N} \mathrm{~d} \rho \rho^{r-(d / 2)} e^{-\gamma \epsilon^{2} \rho} \\
& \times\left(\frac{1}{\sinh (\epsilon \rho / 4 \pi)}-\frac{4 \pi}{\epsilon \rho}\right)
\end{aligned}
$$

the substitution $z=\ln (\epsilon u)$-for the specific case $r=2$ (and $d=4$ )-leads to

$$
\begin{aligned}
I_{b 3}(r=2)= & \lim _{N \rightarrow \infty}-\frac{\epsilon^{-4 \pi \gamma \epsilon}}{16 \pi^{2}} \int_{u_{0}}^{u_{N}} \mathrm{~d} u u^{-1-4 \pi \gamma \epsilon} \\
& \times\left(\frac{1}{\sinh (\ln \epsilon u)}-\frac{1}{\ln \epsilon u}\right)
\end{aligned}
$$

with

$$
u_{0}=\frac{e^{\epsilon / 4 \pi}}{\epsilon}, \quad u_{N}=\frac{e^{N / 4 \pi}}{\epsilon} .
$$

The integral can be performed analytically:

$$
\begin{aligned}
I_{b 3}(r=2)= & \frac{1}{16 \pi^{2}}\left\{\mathcal{B}\left(e^{-N / 2 \pi} ; \frac{1}{2}+2 \pi \gamma \epsilon, 0\right)\right. \\
& -\mathcal{B}\left(e^{-\epsilon / 2 \pi} ; \frac{1}{2}+2 \pi \gamma \epsilon, 0\right)-\mathcal{E}\left(-\gamma \epsilon^{2}\right) \\
& +\mathcal{E}(-\gamma N \epsilon)\}
\end{aligned}
$$

where the incomplete beta function and the exponential integral function, respectively, are defined as

$$
\begin{aligned}
\mathcal{B}(z ; a, b) & =\int_{0}^{z} \mathrm{~d} x x^{a-1}(1-x)^{b-1}, \\
\mathcal{E}(z) & =-\int_{-z}^{\infty} \mathrm{d} x \frac{e^{-x}}{x} .
\end{aligned}
$$

Expanding $I_{b 3}$ in $\epsilon$, one notices that only the second and third expressions in Eq. (C18) lead to $\epsilon$ divergences. Concretely, we obtain a logarithmic divergence:

$$
I_{b 3}(r=2)=-\frac{1}{16 \pi^{2}} \ln \epsilon+\mathcal{O}\left(\epsilon^{0}\right) .
$$

Collecting results, the divergences in the function $\tilde{g}_{2}$ in the weak magnetic field limit are

$$
\begin{aligned}
\tilde{g}_{2}= & -\frac{1}{16 \pi^{3 / 2} \sqrt{\gamma}} \frac{1}{\epsilon}-\frac{\sqrt{2}-4}{32 \pi} \zeta\left(\frac{3}{2}\right) \frac{1}{\sqrt{\epsilon}}-\frac{1}{16 \pi^{2}} \ln \epsilon \\
& +\mathcal{O}\left(\epsilon^{0}\right) .
\end{aligned}
$$

The quark condensate in the chiral limit, according to Eqs. (3.16) and (3.18), features the series

$$
\mathcal{S}[\tilde{g}]=-\hat{c} \epsilon^{2} \tilde{g}_{2}+\frac{\hat{c}^{2} \epsilon^{4}}{2 !} \tilde{g}_{3}-\frac{\hat{c}^{3} \epsilon^{6}}{3 !} \tilde{g}_{4}+\mathcal{O}\left(\tilde{g}_{5}\right),
$$

where

$$
\hat{c}=4 \pi T^{2} \gamma, \quad \gamma=\frac{\bar{l}_{6}-\bar{l}_{5}}{12 \pi} t^{2}
$$


According to Eq. (C14), the leading divergence in the functions $\tilde{g}_{r}$ is proportional to $\epsilon^{3-2 r}$. Therefore each term in the above series gives rise to a contribution linear in $\epsilon$. All these terms have to be taken into account at the order we are operating. The series can be resummed with the result

$$
\mathcal{S}[\tilde{g}]=\frac{\sqrt{2}-1}{2 \sqrt{\pi}} \sqrt{\gamma} \epsilon T^{2} .
$$

The quark condensate in the chiral limit-see Eqs. (3.16) and (3.18) - furthermore involves the other type of Bose functions $g_{r}(M, T, 0)$. The structure of the expansion in the mass parameter $M$ for the specific function $g_{0}(M, T, 0)$ has been analyzed in Refs. [44,46] with the outcome

$$
\begin{aligned}
g_{0}(M, T, 0)= & T^{4}\left[\frac{\pi^{2}}{45}-\frac{1}{12} \frac{M^{2}}{T^{2}}+\frac{1}{6 \pi} \frac{M^{3}}{T^{3}}\right. \\
& +\frac{\left(2 \gamma_{E}-\frac{3}{2}\right)}{32 \pi^{2}} \frac{M^{4}}{T^{4}}+\frac{1}{32 \pi^{2}} \frac{M^{4}}{T^{4}} \ln \frac{M^{2}}{16 \pi^{2} T^{2}} \\
& +2 \pi^{3 / 2} \sum_{n=3}^{\infty} \frac{(-1)^{n}}{n !}\left(\frac{M}{2 \pi T}\right)^{2 n} \\
& \left.\times \Gamma\left(n-\frac{3}{2}\right) \zeta(2 n-3)\right] \quad(T \gg M) .
\end{aligned}
$$

With the recursion relation

$$
g_{r+1}=-\frac{\mathrm{d} g_{r}}{\mathrm{~d} M^{2}},
$$

the series for any other $g_{r}$ with $r=1,2,3, \ldots$ can be derived.

In our case of interest, the relevant mass in these functions is $M_{H}$ :

$$
M_{H}^{2}=\frac{\bar{l}_{6}-\bar{l}_{5}}{48 \pi^{2}} \frac{|q H|^{2}}{F^{2}}=\frac{16 \pi^{2}}{3}\left(\bar{l}_{6}-\bar{l}_{5}\right) t^{4} F^{2} \epsilon^{2},
$$

i.e., the mass of the charged pion that survives the chiral limit. We then find that the leading $\epsilon$ divergences in these functions are

$g_{r}=\frac{(2 r-5) ! ! \gamma^{3 / 2-r}}{2^{3 r-2} \pi^{r-1 / 2}} \epsilon^{3-2 r} T^{4-2 r}, \quad r=2,3, \ldots$
The series of kinematical functions $g_{r}$, as it occurs in the quark condensate,

$$
\mathcal{S}[g]=-\hat{c} \epsilon^{2} g_{2}+\frac{\hat{c}^{2} \epsilon^{4}}{2 !} g_{3}-\frac{\hat{c}^{3} \epsilon^{6}}{3 !} g_{4}+\mathcal{O}\left(g_{5}\right),
$$

hence yields an infinite number of terms that are all linear in $\epsilon$. Resumming, we obtain

$$
\mathcal{S}[g]=\frac{1-\sqrt{2}}{2 \sqrt{\pi}} \sqrt{\gamma} \epsilon T^{2} .
$$

This just cancels the contribution from $\mathcal{S}[\tilde{g}]$ [Eq. (C24)] such that there are no terms linear in $\epsilon$ in the quark condensate coming from here. The logarithmic contributions, however, that are present both in $g_{2}$ and $\tilde{g}_{2}$ do not cancel: in the sum we have

$$
g_{2}+\tilde{g}_{2}=\frac{1}{16 \pi^{2}} \ln \epsilon+\mathcal{O}\left(\epsilon^{-1 / 2}\right),
$$

giving rise to a contribution $\epsilon^{2} \ln \epsilon$ in the quark condensate.

Finally, the $\epsilon$ expansion for the functions $g_{1}(0, T, 0)$ and $\tilde{g}_{1}(0, T, H)$ that also appear in the quark condensate [Eqs. (3.16) and (3.18)] has been provided in Refs. [11,44]. For completeness we quote the result:

$$
\begin{aligned}
g_{1}(0, T, 0)= & \frac{1}{12} T^{2} \\
\tilde{g}_{1}(0, T, H)= & -\left\{\frac{\left|I_{1 / 2}\right|}{8 \pi^{3 / 2}} \sqrt{\epsilon}-\frac{\ln 2}{16 \pi^{2}} \epsilon+\frac{\zeta(3)}{384 \pi^{4}} \epsilon^{2}\right. \\
& \left.-\frac{7 \zeta(7)}{98304 \pi^{8}} \epsilon^{4}+\mathcal{O}\left(\epsilon^{6}\right)\right\} T^{2},
\end{aligned}
$$

with

$I_{1 / 2}=\int_{0}^{\infty} \mathrm{d} \rho \rho^{-1 / 2}\left(\frac{1}{\sinh (\rho)}-\frac{1}{\rho}\right) \approx-1.516256$.
[1] I. A. Shushpanov and A. V. Smilga, Phys. Lett. B 402, 351 (1997).

[2] N. O. Agasian and I. A. Shushpanov, Phys. Lett. B 472, 143 (2000).

[3] N. O. Agasian, Phys. Lett. B 488, 39 (2000).
[4] N. O. Agasian, Phys. At. Nucl. 64, 554 (2001).

[5] N. O. Agasian and I. A. Shushpanov, J. High Energy Phys. 10 (2001) 006.

[6] T. D. Cohen, D. A. McGady, and E. S. Werbos, Phys. Rev. C 76, 055201 (2007). 
[7] E. S. Werbos, Phys. Rev. C 77, 065202 (2008).

[8] N. O. Agasian, Phys. At. Nucl. 71, 1967 (2008).

[9] J. O. Andersen, Phys. Rev. D 86, 025020 (2012).

[10] J. O. Andersen, J. High Energy Phys. 10 (2012) 005.

[11] C. P. Hofmann, Phys. Rev. D 99, 014030 (2019).

[12] M. D'Elia, S. Mukherjee, and F. Sanfilippo, Phys. Rev. D 82, 051501 (2010).

[13] M. D'Elia and F. Negro, Phys. Rev. D 83, 114028 (2011).

[14] G. S. Bali, F. Bruckmann, G. Endrödi, Z. Fodor, S. D. Katz, S. Krieg, A. Schäfer, and K. K. Szabó, J. High Energy Phys. 02 (2012) 044.

[15] V. V. Braguta, P. V. Buividovich, T. Kalaydzhyan, S. V. Kuznetsov, and M. I. Polikarpov, Phys. At. Nucl. 75, 488 (2012).

[16] G. S. Bali, F. Bruckmann, G. Endrödi, Z. Fodor, S. D. Katz, and A. Schäfer, Phys. Rev. D 86, 071502 (2012).

[17] G. S. Bali, F. Bruckmann, M. Constantinou, M. Costa, G. Endrödi, Z. Fodor, S. D. Katz, H. Panagopoulos, and A. Schäfer, Phys. Rev. D 86, 094512 (2012).

[18] V. G. Bornyakov, P. V. Buividovich, N. Cundy, O. A. Kochetkov, and A. Schäfer, Phys. Rev. D 90, 034501 (2014).

[19] E.-M. Ilgenfritz, M. Müller-Preussker, B. Petersson, and A. Schreiber, Phys. Rev. D 89, 054512 (2014).

[20] M. D’Elia, F. Manigrasso, F. Negro, and F. Sanfilippo, Phys. Rev. D 98, 054509 (2018).

[21] G. Endrödi, M. Giordano, S. D. Katz, T. C. Kovács, and F. Pittler, J. High Energy Phys. 07 (2019) 007.

[22] R. Gatto and M. Ruggieri, Phys. Rev. D 83, 034016 (2011).

[23] A. Amador and J. O. Andersen, Phys. Rev. D 88, 025016 (2013).

[24] M. Ferreira, P. Costa, D. P. Menezes, C. Providencia, and N. N. Scoccola, Phys. Rev. D 89, 016002 (2014).

[25] R. L. S. Farias, K. P. Gomes, G. Krein, and M. B. Pinto, Phys. Rev. C 90, 025203 (2014).
[26] E. J. Ferrer, V. de la Incera, I. Portillo, and M. Quiroz, Phys. Rev. D 89, 085034 (2014).

[27] M. Ferreira, P. Costa, O. Lourenco, T. Frederico, and C. Providencia, Phys. Rev. D 89, 116011 (2014).

[28] M. Ferreira, P. Costa, and C. Providencia, Phys. Rev. D 90, 016012 (2014).

[29] R. Zhang, W. Fu, and Y. Liu, Eur. Phys. J. C 76, 307 (2016).

[30] S. Nam and C.-W. Kao, Phys. Rev. D 83, 096009 (2011).

[31] M. Frasca and M. Ruggieri, Phys. Rev. D 83, 094024 (2011).

[32] F. Bruckmann, G. Endrödi, and T. G. Kovács, J. High Energy Phys. 04 (2013) 112.

[33] V. D. Orlovsky and Y. A. Simonov, J. High Energy Phys. 09 (2013) 136.

[34] G. Colucci, E. S. Fraga, and A. Sedrakian, Phys. Lett. B 728, 19 (2014).

[35] V. D. Orlovsky and Y. A. Simonov, Phys. Rev. D 89, 074034 (2014).

[36] A. Haber, F. Preis, and A. Schmitt, Phys. Rev. D 90, 125036 (2014).

[37] N. Mueller and J. M. Pawlowski, Phys. Rev. D 91, 116010 (2015).

[38] H. Leutwyler, in Hadron Physics 94-Topics on the Structure and Interaction of Hadronic Systems, edited by V.E. Herscovitz, C. A. Z. Vasconcellos and E. Ferreira (World Scientific, Singapore, 1995), p. 1.

[39] S. Scherer, Adv. Nucl. Phys. 27, 277 (2003).

[40] C. P. Hofmann, Phys. Rev. D 101, 114031 (2020).

[41] J. Bijnens, G. Colangelo, and G. Ecker, Ann. Phys. (N.Y.) 280, 100 (2000).

[42] J. Gasser and H. Leutwyler, Ann. Phys. (N.Y.) 158, 142 (1984).

[43] S. Aoki et al., Eur. Phys. J. C 80, 113 (2020).

[44] P. Gerber and H. Leutwyler, Nucl. Phys. B321, 387 (1989).

[45] S. Weinberg, Physica (Amsterdam) 96A, 327 (1979).

[46] C. P. Hofmann, Phys. Rev. B 81, 014416 (2010). 